\title{
Evaporation of closely-spaced interacting droplets arranged in a single row
}

\author{
G. Castanet*, L. Perrin, O. Caballina, F. Lemoine \\ LEMTA, UMR 7563, Université de Lorraine, Vandœuvre-Lès-Nancy 54518, France \\ LEMTA, UMR 7563, CNRS, Vandœuvre-Lès-Nancy 54518, France
}

Keywords:

Droplet evaporation

Interacting droplets

Sprays

Fuel droplet

\begin{abstract}
A B S T R A C T
Predictive models of heating and evaporation of fuel droplets in the dense region of sprays are essential to optimize the design of combustion chambers in internal combustion engines. This topic is addressed here, based on an experimental study using lines of equally-spaced droplets evaporating in a high temperature chamber $\left(540{ }^{\circ} \mathrm{C}\right)$. The experimental set-up allows controlling several key parameters including the droplet size, velocity and the inter-droplet distance. The volume-averaged temperature of the droplets is measured using two-color laser-induced fluorescence, while the droplet size and velocity are deduced from a double-pulse shadowgraphy method. The combination of these measurement techniques allows evaluating the heating and evaporation rates of single-component droplets made of ethanol, $n$-dodecane, $n$-decane and isohexane. Nusselt and Sherwood numbers are estimated from the experiments and compared with the existing correlation concerning the isolated droplet, which allows quantifying the effects of droplet interactions on the heat and mass transfers. The droplet spacing appears to have a strong influence on the size and temperature evolutions. However, it also seems necessary to consider the development of a boundary layer around the chain of droplets. As the thickness of the boundary layer increases with the distance from the injector, forced convection has a more and more limited influence on the heat and mass transport. Inside the boundary layer, the transfers are mainly governed by diffusion and convection by the Stefan flow. In a first approach, these effects are partially incorporated in a reduced parameter using the concept of volume of influence. Then, a more detailed study based on numerical simulation is carried out. The Navier-Stokes equations and the vapor transport equation are solved for various periodical arrangements of droplets. A parametric study of the influence of the main characteristic numbers involved allows to infer a correlation for Nusselt and Sherwood numbers which is finally validated against the measurements.
\end{abstract}

\section{Introduction}

Predictive models of heating and evaporation of fuel droplets in the dense spray region, i.e. when droplet-to-droplet interactions play a dominant role, are essential to optimize the design of the combustion chambers in internal combustion engines. In a spray, the complex trajectories of the droplet, their dispersion in size and velocities, and the non-uniform spatial distribution of the droplet number density make difficult to separate the influence of the different parameters for the analysis of the phenomena. For this reason, fundamental studies, either experimental, theoretical or numerical, are generally based on simplified configurations, like free falling individual droplets, monodisperse droplet lines, and arrays of monodisperse droplet streams.
Labowsky [1], Sangiovanni and Labowsky [2], Marberry et al. [3] studied extensively the interactions between the droplets in the case of monodisperse droplet streams. To describe the influence of the droplet interactions on the evaporation rate and the drag coefficient, they introduced the spacing parameter, denoted $C$ hereafter, which is defined as the ratio between the inter-droplet distance $L$ and the droplet diameter $d$. Virepinte et al. [4] investigated experimentally the evaporation of periodically-arranged monosized ethanol droplets which are injected upward in a flame supplied by the fuel vapor released by the vaporizing droplets. They showed that the rate of evaporation of the isolated drop should be corrected to match their experiments. Also, they established a correction based on the spacing parameter $C$. The same experimental configuration was also studied by Castanet et al. [5] who measured at the same time the droplet size and temperature and proposed a correction for both the Sherwood and Nusselt numbers based on the spacing parameter. Using the same 


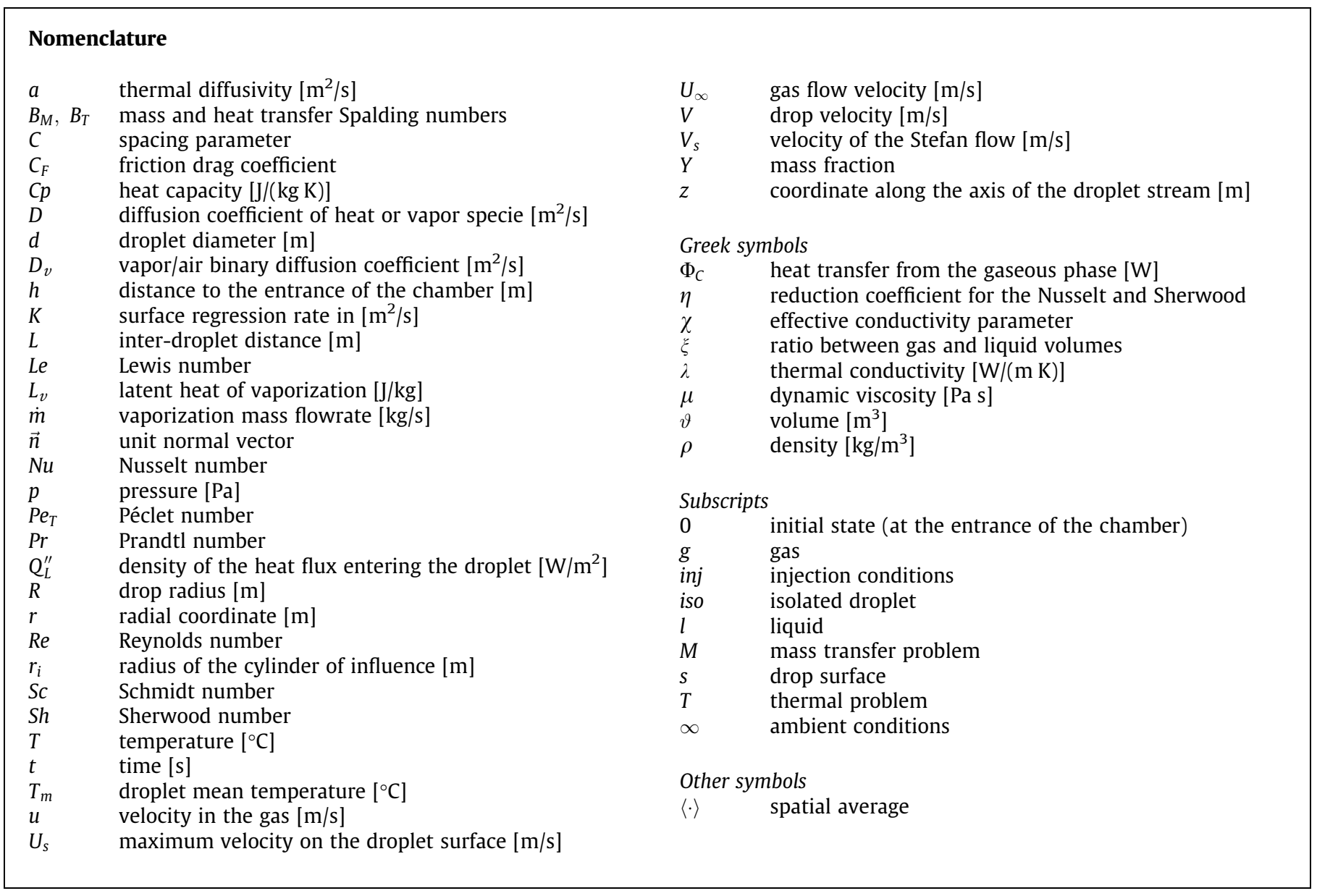

approach, Castanet et al. [6] carried out a study of the vaporization rate of a monodisperse chain in the thermal boundary layer of a heated plate oriented vertically. The above-mentioned experimental studies [4-6] were limited to droplets made of ethanol and the spacing parameter $C$ was the only parameter considered to evaluate the reduction of the Nusselt and Sherwood numbers compared to an equivalent isolated droplet of the same size, moving at the same velocity in the same free stream. Deprédurand et al. [7] studied the evaporation of droplets made of different fuels and demonstrated that the distance parameter was not sufficient to describe the reduction of heat and mass transfers compared to the isolated droplet. They showed that the volatility of the fuels (related to the velocity of the Stefan flow) plays also an important role. To account for the effects of droplet interactions, they suggested using a dimensionless time defined as the ratio of the characteristic time of advection through the resistive film introduced by Abramzon and Sirignano [8] and the time between two consecutive droplets at a given location. Based on this dimensionless time, Deprédurand et al. [7] proposed an empirical correlation for the reduction of the Nusselt and Sherwood numbers of interacting monosized droplets arranged in single row. However, their experiments were limited to moderately high ambient temperatures and therefore it was difficult to observe significant evaporation for the low-volatile fuels and significant temperature rise for the highly volatile fuels, which hindered their estimations of Nusselt and Sherwood numbers.

Imaoka and Sirignano [9] developed advanced modeling of the evaporation and combustion of complex 3D arrangements of droplets. For unitary Lewis numbers, they investigated 3D arrays having symmetrical or asymmetrical configurations. In the case of an asymmetric array, they demonstrated that the reduction of the evaporation rate compared to an isolated droplet scales with the ratio between the volume of gas in the array and the liquid volume of the droplets included in the array. They showed that the vaporization rate is all the more reduced that this volume ratio is weak. However, their study was limited to the case where forced and natural convection are not important. The gas motion relative to droplets is caused by Stefan convection only. As a consequence, the computations can be reduced to the resolution of a Laplace's equation in the gas which is relatively simple to perform even for complex geometrical arrangements of droplets. Chiang and Sirignano [10] were one of the first to investigate numerically the evaporation of interacting droplets moving relatively to the carrier gas by solving the Navier-Stokes equations. However their investigations were limited to two or three droplets moving in tandem. More recently, the transient convective burning and evaporation of droplets arranged in single-layer arrays and subjected to an hot air stream was investigated by $\mathrm{Wu}$ and Sirignano [11] solving the Navier-Stokes, energy and species equations. Droplet regression, deceleration of the stream flow due to the drag of the droplets, internal circulation, variable properties, non-uniform surface temperature, and surface tension are considered in these simulations. By setting boundary periodical conditions in the calculations, $\mathrm{Wu}$ and Sirignano [11] were able to simulate semiinfinite periodic arrays and infinite periodic arrays. Castanet et al. [12] also simulated infinite droplet arrays using boundary periodical conditions for a limited number of cases and did the comparison with measurements of the temperature field inside burning droplets. Their results show the importance of droplets interactions as well as the Marangoni effect to describe the droplet heating. 
Despite the aforementioned studies, predicting the effect of interactions between closely-spaced droplets remains an opened issue [13]. The present study focuses on the characterization of the processes that affect the heat and mass transfers in a chain of monodisperse droplets. To that end, an experimental study is carried out using a system of droplet generation that allows a fine control of the size, velocity and temperature of the droplets as well as the distance between them. Droplets are injected in a chamber where a very high ambient temperature $\left(540^{\circ} \mathrm{C}\right)$ and a steady laminar flow are maintained. The volume-averaged temperature of the droplets is measured by the two-color laser-induced fluorescence thermometry and the droplet size and velocity by shadowgraphy [14]. These two techniques allow quantifying the heating and evaporation rates of single-component droplets made of $n$-dodecane, $n$-decane, ethanol and isohexane. An inverse method is developed to estimate the Nusselt and Sherwood numbers from the size and temperature measurements. Results of these estimations reveal that the reduction of Nusselt and Sherwood numbers relative to the isolated droplet is not well correlated with the spacing parameter. A better correlation can be obtained by introducing a reduced variable derived from the concept of volume of influence. As this variable not only depends on the droplet spacing but also on the droplet velocity, the vapor diffusivity and the velocity of the Stefan flow, this indicates that other parameters than the geometry must be invoked to describe the droplet interactions. Finally, a more detailed study based on numerical simulation is carried out. The Navier-Stokes equations and the vapor transport equation are solved for various periodical arrangements of droplets. A parametric study of the main characteristic numbers involved in the problem allows to infer a correlation for Nusselt and Sherwood numbers which is finally validated against the measurements.

\section{Experimental set-up and measurement techniques}

\subsection{The evaporation chamber and experimental facilities}

A piezoelectric droplet generator is used to produce a chain of monodisperse droplets. The liquid jet coming out of the injector is disintegrated into mono-sized droplets for specific vibration frequencies of the piezoceramic attached to the injector body. Then, the droplets are injected into an enclosure supplied with hot air (up to $540{ }^{\circ} \mathrm{C}$ ) produced by an electrical heater (Fig. 1). The gas flow ensures an almost uniform temperature field inside the enclosure and also prevents fuel vapor accumulation. In order to limit the air cooling due to the heat conduction inside the wall of the chamber, the outer envelope of the enclosure is covered with a thick thermal insulation layer. Additionally, a resistive electrical

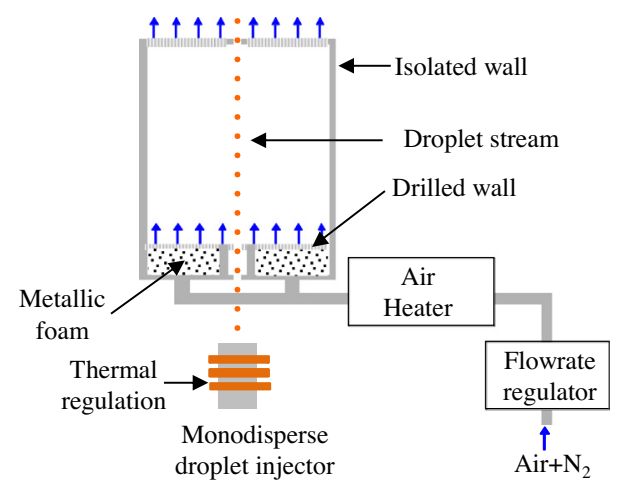

Fig. 1. Schema of the experimental set-up showing the heated enclosure and the generation of a droplet monodisperse stream. wire is inserted within the wall to regulate the wall at the same temperature as the incoming air. Nitrogen is added to the air to reduce the fraction of oxygen in the gas mixture and thus avoid unwanted ignition of the fuel vapor. The gas flow is quieted at the entrance of the chamber by passing through a layer of metallic foam and a drilled wall. The gas velocity remains small (about 0.1$0.3 \mathrm{~m} / \mathrm{s}$ ) compared to that of the droplets (typically a few $\mathrm{m} / \mathrm{s}$ ). Quartz windows managed in the wall of the enclosure provide several optical accesses to operate optical diagnostics. Three parameters are measured along the monodisperse droplet stream: the temperature, the diameter and the velocity of the droplets (Fig. 2).

\subsection{Temperature measurements}

The droplet temperature is measured by the two-color laserinduced fluorescence (2cLIF) thermometry, initiated by Lavieille et al. [15]. This technique consists in seeding the liquid fuel by a fluorescent dye, here pyrromethene 597-C8, which is easily soluble in alkanes and alcohols. The fluorescence of pyrromethene 597-C8 is induced by a frequency-doubled CW Nd-Yag laser at $532 \mathrm{~nm}$. The fluorescence quantum yield of the dye is fairly sensitive to the temperature, which offers the possibility to determine the liquid temperature from the measurement of the intensity of the fluorescence signal. One difficulty for the measurements relates to the fact that the shape and amplitude of the fluorescence signal emitted from a droplet depend on many factors which can be hardly controlled such as the size of the droplet compared to the diameter of the laser beam and the droplet trajectory [16]. For instance, the fluorescence signal changes drastically if the droplet is off-centered relatively to the laser beam and/or the focal point of the detection optics. The problem of this multiple-dependency of the LIF signal can be overcome for some dyes like pyrromethene 597-C8 by using intensity ratios. Because the sensitivity in temperature varies among the wavelengths that compose the fluorescence spectrum, the signal ratio between two spectral bands is temperaturedependent. The fluorescence ratio allows eliminating the dependence on unknown factors among others: the local illumination by the incident laser light, the dye concentration in the droplet, and the dimensions of the measurement volume. In the case of pyrromethene 597-C8, two spectral bands having very different temperature sensitivity can be found allowing a good accuracy in the measurements (about $\pm 1^{\circ} \mathrm{C}$ ). For each of the liquid fuels, a calibration of the fluorescence ratio as a function of temperature is performed in a cell where the liquid is perfectly stirred and controlled in temperature.

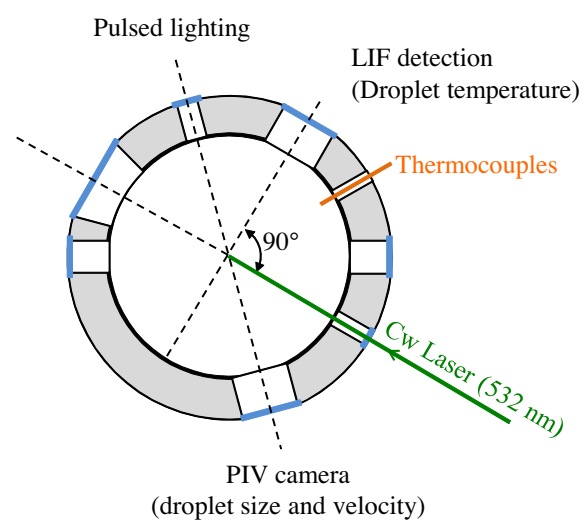

Fig. 2. Optical accesses through the wall of the enclosure for the implementation of the different optical techniques. 


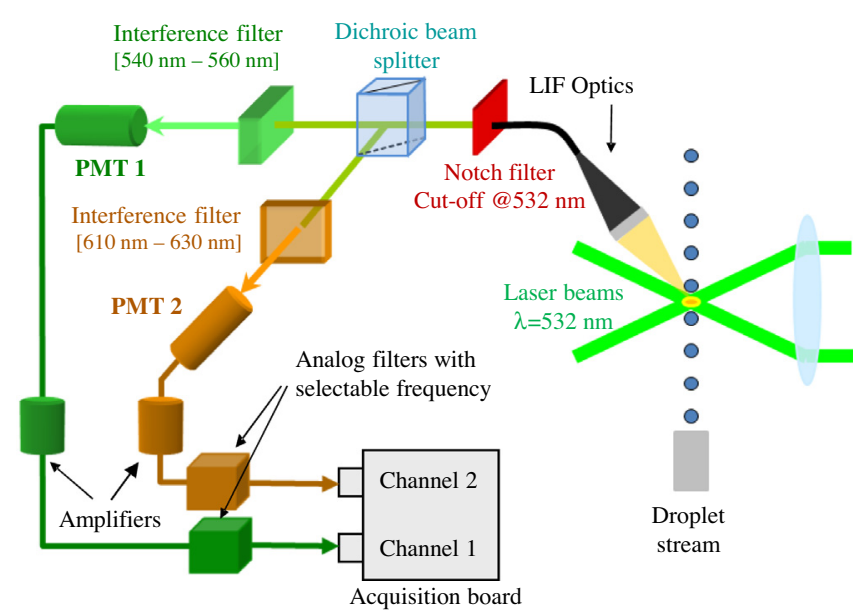

Fig. 3. Schematic view of the 2 cLIF system used for the measurement of the droplet temperature.

The measurement system depicted in Fig. 3 is in all points similar to that used by Perrin et al. [14]. As featured in Fig. 3, two intersecting laser beams are used to form the probe volume. Strictly speaking, this arrangement is not necessary, but turns out to be very useful in practice. The optics are arranged once and for all, so that the intersection of the laser beams coincides with the region observed by the LIF detector. Hence, even though the trajectory of the droplets changes from one experiment to another, the optical alignment is very easy to make. The only remaining adjustment is to move the droplet stream as a whole (the injector being placed on a 3D translation stage) until the droplets pass through the intersection of the laser beams, which is easy to control visually. The intersection of the laser beams also serves as a reference target for the positioning of the camera utilized to determine the size and velocity of the droplets with the technique described in Section 2.3 .

A particular attention was paid to suppress the morphological dependent resonances (MDRs), which lead to light amplification at some preferential wavelengths in the low-absorbed regions of the fluorescence spectrum. As discussed by Perrin et al. [14], even though MDRs can be sometimes rather difficult to detect, they generally lead to significant measurement errors. A small volume of a non-fluorescent dye, namely Oil Blue $\mathrm{N}$, is added to the liquid fuel (on the order of a few $\mu \mathrm{g} / \mathrm{L}$ ). The strong absorption of Oil Blue $\mathrm{N}$ at wavelengths, which are not or very weakly absorbed by pyrromethene 597-C8, prevents light amplification within the droplets from occurring.

An other point that is important to mention is that the measured temperature is not exactly the volume-averaged temperature of the droplet. Taken into account the configuration of their measurement system (focal lengths of the lenses, sizes of the laser beam and the droplets,...), Perrin et al. [14] estimated that the measured temperature $T_{L I F}$ can be well approximated by $T_{\text {LIF }} \approx\left(T_{c}+T_{m}\right) / 2$, where $T_{c}$ is the temperature at the center of the droplet and $T_{m}$ is the actual mean temperature of the droplet. This approximation will be used hereafter to compare the measured temperatures with those obtained from the models. However, it is only valid for the present experiments.

\subsection{Size and velocity measurements}

The size and the velocity of the droplets were measured by shadowgraphy as described by Perrin et al. [14]. The backlighting of the droplets is achieved by a double cavity PIV laser focused on a PMMA sample doped with a fluorescent dye (Fig. 4). The fluorescence

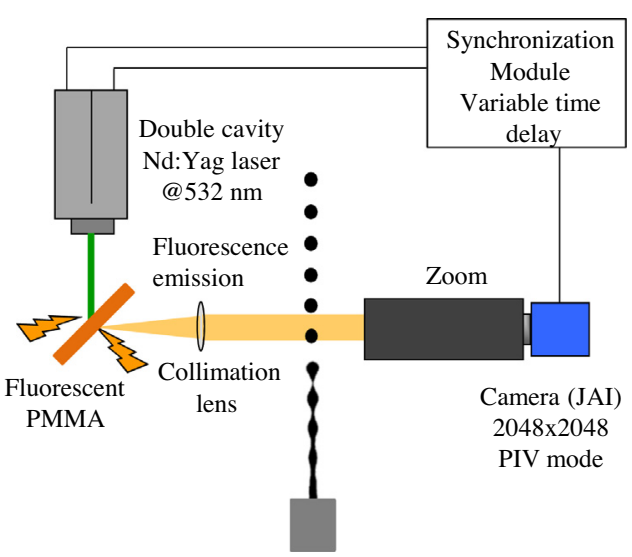

Fig. 4. Optical system for the measurement of the droplet diameter and velocity by shadowgraphy.

emitted by the PMMA plate is collimated and used as a backlighting source, which avoid the problem of the speckles occurring with laser light. The short duration of the laser pulses and the short lifetime of the fluorescence allow to freeze the droplet motion in the images. Shadow images are recorded by a PIV camera. The velocity of the droplets is determined from their displacement within a pair of images. The camera $(2048 \times 2048$ pixels $)$ is equipped with a high magnification zoom which yields a resolution on the order of $1 \mu \mathrm{m} / \mathrm{pxl}$. The absolute accuracy of the drop sizing is therefore about $1 \mu \mathrm{m}$, but the relative accuracy within a set of measurements is much better (typically less than $0.1 \mu \mathrm{m}$ ). Images are also corrected from the pincushion distortion induced by the zoom lens. The periodicity of the droplet chain makes particularly easy the determination of the time elapsed from the entrance inside the evaporation chamber. Provided the velocity profile $V(z)$ measured along the droplet stream ( $z$-axis), the time taken by the droplets to cover a distance $h$ is given by:

$t=\int_{0}^{h} \frac{d z}{V(z)}$

\section{General phenomena}

When a droplet penetrates into an overheated environment, a part of the heat it receives is used to evaporate the liquid at the droplet surface, while the rest is transferred inside the droplet contributing to the raise of the droplet temperature. Among other things, the heat fluxes associated with these two contributions depends on the liquid properties and the spacing between the droplets as illustrated in the following paragraphs.

\subsection{Comparison between the fuels}

In a first stage, the afore-described measurement techniques were used to compare the evaporation and heating of droplets of different fuels, namely ethanol, iso-hexane, $n$-decane and $n$ dodecane. Fig. 5 shows the time evolution of the temperature and the squared diameter in the case of droplets injected under similar conditions. The spacing parameter and the velocity are almost the same at the entrance of the chamber (here $C_{0}=4.7$ and $V_{0}=11.8 \mathrm{~m} / \mathrm{s}$ ), but the temperature and the size of the droplets can be slightly different from one experiment to another. For this reason, it was preferred to display in Fig. 5b the increase in temperature as a function of the characteristic time of heat diffusion within the droplet, i.e. $a_{l} \cdot t / d_{0}^{2}$ where $a_{l}$ is the liquid thermal 
(a)

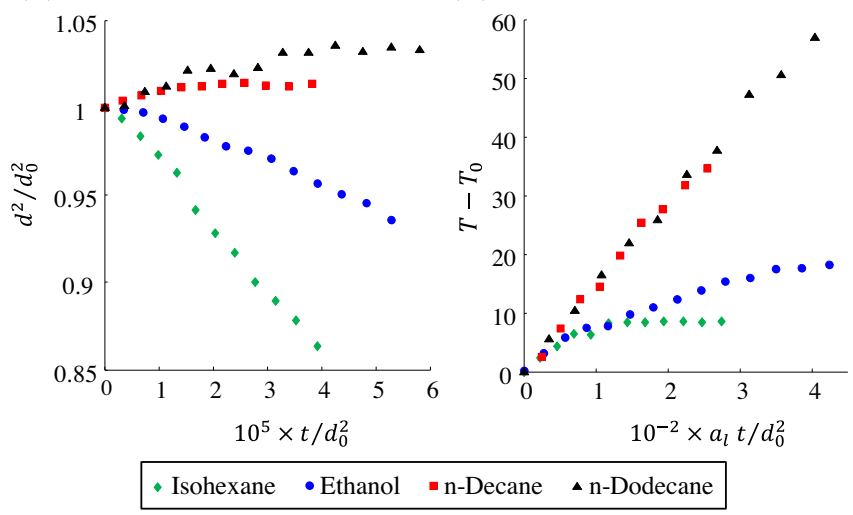

Fig. 5. Evolution of the size and temperature of droplets made of different fuels (Ethanol: $d_{0}=111.6 \mu \mathrm{m}, T_{0}=35.1^{\circ} \mathrm{C}$; Isohexane: $d_{0}=109 \mu \mathrm{m}, T_{0}=28.3^{\circ} \mathrm{C} ; n$ Dodecane: $d_{0}=109 \mu \mathrm{m}, T_{0}=25.1{ }^{\circ} \mathrm{C}$; $n$-Decane: $d_{0}=109.7 \mu \mathrm{m}, T_{0}=34.5^{\circ} \mathrm{C}$, and for all the cases $C_{0}=4.7$ and $V_{0}=11.8 \mathrm{~m} / \mathrm{s}$ )

diffusivity. In Fig. 5a, both the time and the squared diameter are divided by $d_{0}^{2}$.

For highly volatile fuels, namely isohexane and ethanol, the droplet temperature reaches rapidly an equilibrium. It should be emphasized that the equilibrium temperature is significantly lower than the boiling temperature of these fuels. The equilibrium temperature is about $40^{\circ} \mathrm{C}$ for isohexane and $50{ }^{\circ} \mathrm{C}$ for ethanol, while the boiling temperatures of these fuels are respectively $60^{\circ} \mathrm{C}$ and $78^{\circ} \mathrm{C}$. The equilibrium temperature corresponds to the wet-bulb temperature of the fuel at the ambient conditions in the chamber $\left(T_{\infty}=540{ }^{\circ} \mathrm{C}\right.$ and $\left.Y_{\infty}=0\right)$. After having reached a thermal equilibrium, the heat transferred to the droplet from the gas phase is entirely used for liquid vaporization. A linear decrease of the droplet surface is then clearly observed in Fig. 5a. For the less volatile fuels, namely $n$-decane and $n$-dodecane, the droplet evolution is far different. Contrary to the volatile fuels, no size reduction can be pointed out. Instead, the droplet size increases due to the fact that evaporation accounts for less than the thermal expansion of the liquid. The heating proceeds at a fast rate but the time covered by the measurements is not sufficient to reach an equilibrium temperature as for the volatile fuels. All the heat transferred from the gas to the droplet is used for heating up the liquid, which explains why the temperature of $n$-decane and $n$-dodecane droplets (having about the same injection parameters) raises at nearly the same rate in Fig. 5b.

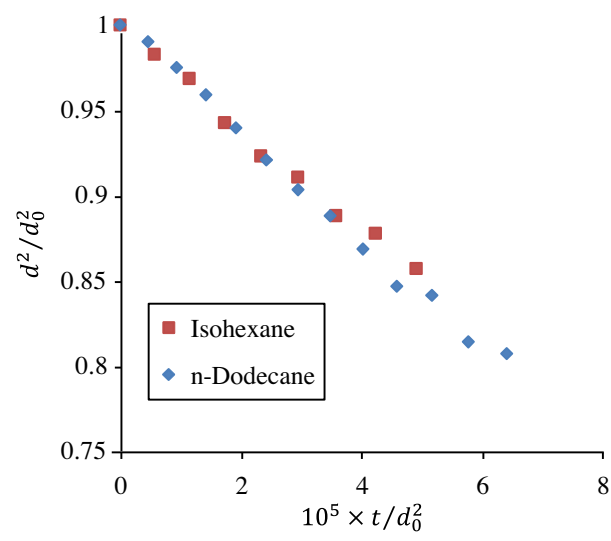

Fig. 6. Time evolution of the droplet size. For $n$-dodecane, the experimental conditions are: $T_{0}=170{ }^{\circ} \mathrm{C}, d_{0}=91.7 \mu \mathrm{m}, V_{0}=12.8 \mathrm{~m} / \mathrm{s}, C_{0}=4.7$, and for isohexane: $T_{0}=33^{\circ} \mathrm{C}, d_{0}=93.3 \mu \mathrm{m}, V_{0}=12.0 \mathrm{~m} / \mathrm{s}, C_{0}=4.5$.
In Fig. 6, the reduction of the squared diameter is compared for isohexane droplets at $T_{0}=33{ }^{\circ} \mathrm{C}$ and $n$-dodecane droplets at the equilibrium temperature $\left(T_{e q} \approx 170^{\circ} \mathrm{C}\right)$ while $C_{0}, d_{0}$ and $V_{0}$ are approximately the same. It is observed that the evaporation rates are very close which illustrates that the injection temperature drastically changes the volatility of a fuel.

\subsection{Influence of the spacing between the droplets}

The interactions between neighbor droplets are known to reduce the heat and mass transfers in comparison to the isolated droplets. This effect can be evidenced in the experiments by changing the spacing between the droplets while maintaining unchanged as much as possible the other injection parameters (size, velocity and temperature of the droplets). Fig. 7 shows a significant decrease of the evaporation rate, defined as $K=-d\left(d^{2}\right) / d t$, with the spacing parameter $C$ in the case of ethanol droplets. The $d^{2}$-law is rather well verified for these droplets which quickly reaches an equilibrium temperature after entering the chamber. Even for a spacing parameter as large as $C=9.2$, the deviation from the isolated droplet remains substantial. The evolution of the isolated drop was simulated by using the model described in Section 3.3 imposing $\eta_{M, T}=1$. The effect of the droplet spacing was also observed in terms of heating. Fig. 8 shows for $n$-dodecane droplets that the heating rate increases with the spacing parameter. Here also, differences with the model of the isolated droplet can be pointed out.

\subsection{Experimental estimation of the heat and mass transfers}

Nusselt and Sherwood numbers, denoted respectively $\mathrm{Nu}$ and $\mathrm{Sh}$, are classically used to evaluate the rate of heat and mass transfers between the droplet and its gaseous environment. The objective of the current section is to show that these numbers can be evaluated from the measurements of the size and the temperature. The resolution of this inverse problem is described in the following. For the direct problem, a model is used to determine the size and temperature evolutions of a droplet subjected to any arbitrary $\mathrm{Nu}$ and Sh. Only a brief summary of this model is presented hereafter as the modeling heavily relies on well-known classical assumptions. In particular, the quasi-steady approximation is made to describe the droplet evaporation $[17,18]$. When a droplet moves into a hot gas environment, it receives a heat flux $\Phi_{c}$ from the gas which can be expressed using the Nusselt number:

$\Phi_{c}=\pi d N u \lambda_{g}\left(T_{\infty}-T_{s}\right)$.

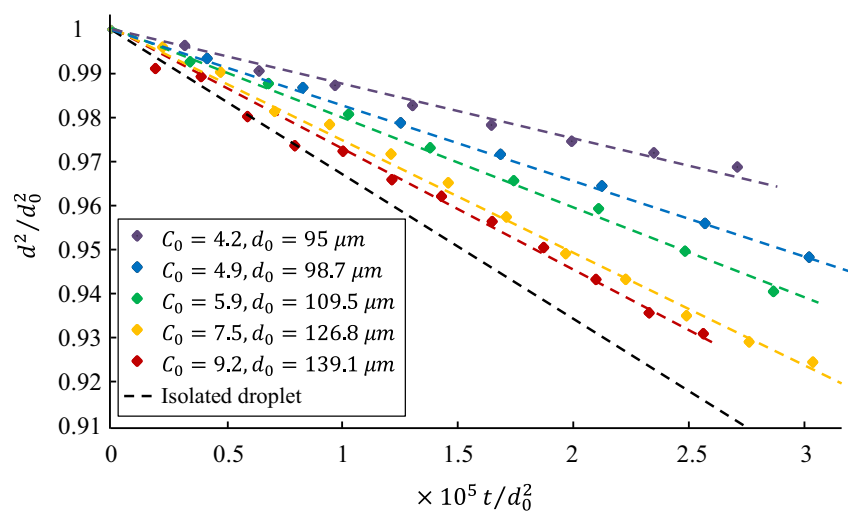

Fig. 7. Influence of the spacing parameter on the evaporation rate of ethanol droplets injected at $V_{0} \approx 13 \mathrm{~m} / \mathrm{s}$ near the equilibrium temperature at $540{ }^{\circ} \mathrm{C}$ and $Y_{\infty}=0$. The size evolution of the isolated droplet was evaluated based on the model presented in Section 3.3 fixing $\eta_{M}=\eta_{T}=1$. 


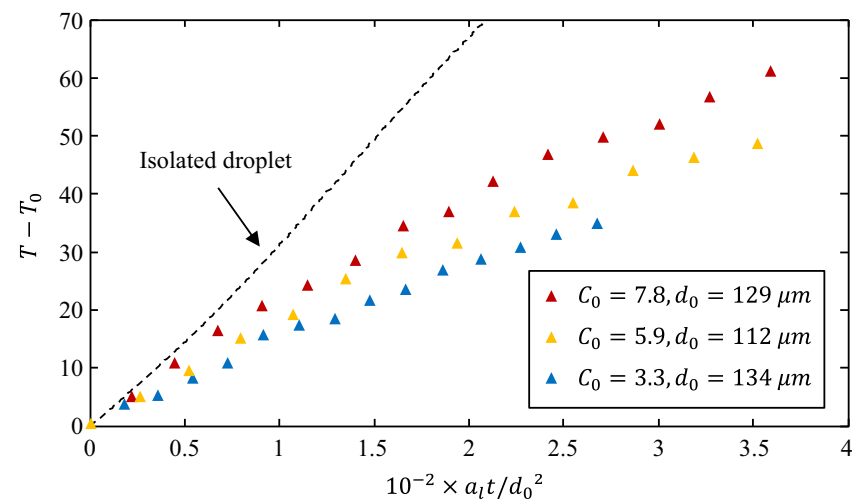

Fig. 8. Influence of the spacing parameter on the heating rate of $n$-dodecane droplets $\left(T_{0} \sim 50^{\circ} \mathrm{C}, V_{0} \approx 13 \mathrm{~m} / \mathrm{s}\right)$. The temperature evolution of the isolated droplet was evaluated based on the model presented in Section 3.3 fixing $\eta_{M}=\eta_{T}=1$.

The resulting instantaneous mass vapor flowrate $\dot{m}$ is given by:

$\dot{m}=\pi d \rho_{g} D_{v} \operatorname{Sh} B_{M}$.

In these expressions, $\rho_{g}$ and $\lambda_{g}$ are the density and the thermal conductivity of the gas, $D_{v}$ is the diffusion coefficient of vapor in air. $B_{M}=\left(Y_{s}-Y_{\infty}\right) /\left(1-Y_{s}\right)$ is the mass transfer Spalding number related to the mass fraction of vapor at the drop surface $\left(Y_{s}\right)$ and in the ambient gas $\left(Y_{\infty}\right) . T_{\infty}$ is the temperature of the ambient gas and $T_{s}$ is the surface temperature of the droplet. In the case of an isolated droplet, Nusselt and Sherwood numbers can be determined from the film theory. Expressions of $N u_{\text {iso }}$ and $S h_{\text {iso }}$ were derived by Abramzon and Sirignano [8] using Eq. (31). In the presence of droplet interactions, these expressions are not valid and two reduction factors $\eta_{T}$ and $\eta_{M}$ are introduced:

$\eta_{T}=\mathrm{Nu} / \mathrm{Nu} u_{\text {iso }}$ and $\eta_{M}=S h / S h_{\text {iso }}$

The factors $\eta_{T}$ and $\eta_{M}$ are the targets of the inversion to be determined from the experimental results. For given value of $\eta_{M, T}$, the droplet evolution can be simulated using the procedure described by Abramzon and Sirignano [8]. The approach of effective thermal conductivity is implemented to take into account the liquid circulation within the droplet induced by the gas frictions at the droplet surface. The effective thermal conductivity $\lambda_{\text {eff }}$ is obtained using the following equation:

$\lambda_{\text {eff }}=\chi\left(P e_{T}\right) \cdot \lambda_{l}$,

and $\chi\left(P e_{T}\right)=1.86+0.86 \tanh \left[2.245 \log _{10}\left(P e_{T} / 30\right)\right]$,

where $P e_{T}=\rho_{l} C p_{l} U_{s} d / \lambda_{l}$ is the thermal Peclet number based on the sliding velocity of the liquid interface $U_{s}$. In the absence of Marangoni effect, gas frictions are the main source of the internal liquid motions. Assuming a velocity field close to that of a Hill vortex, the stress continuity at the droplet surface yields [8]:

$U_{s}=\frac{1}{32}\left(\frac{\mu_{g}}{\mu_{l}}\right) \operatorname{Re} \Delta U_{\infty} C_{F}$,

where $R e=\rho_{\infty} \Delta U_{\infty} d / \mu_{g}$ is the Reynolds number, $\mu_{g}$ the viscosity of the vapor-air mixture and $\Delta U_{\infty}=\left|V-U_{\infty}\right|$ the relative gas/droplet velocity. For interacting droplets, the friction drag coefficient $C_{F}$ is affected by the wake effects in the row of droplets. Therefore, the expression proposed by Abramzon and Sirignano [8] in the case of
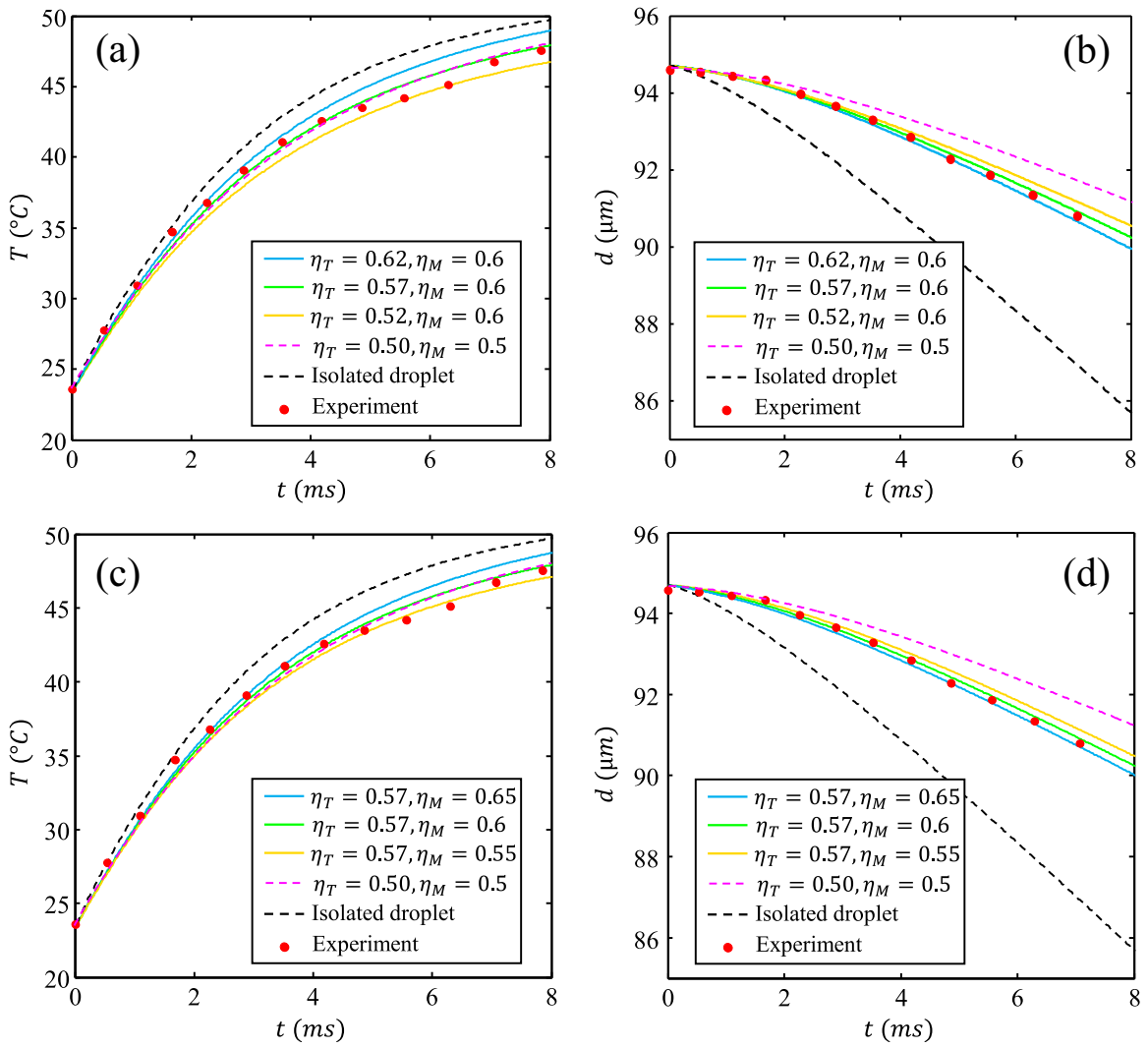

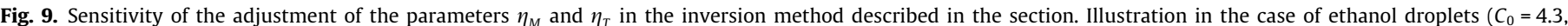

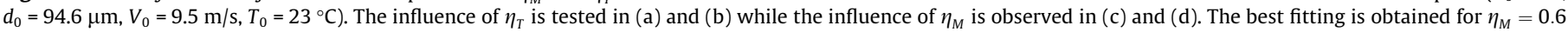
and $\eta_{T}=0.57$. For comparison, the isolated droplet and the couple $\eta_{M}=\eta_{T}=0.5$ are also added. 
an isolated droplet does not applied. Instead, the following approximation can be proposed:

$$
\begin{aligned}
& C_{F}=\frac{12.69 K_{F}}{R e^{2 / 3}\left(1+B_{M}\right)}, \\
& \text { with } \quad K_{F}=0.67[1-\exp (-a-b\{C-1\})]-0.1, \\
& \qquad \begin{array}{l}
a=0.0988+0.00339 R e, \\
\text { and } \quad b=0.0956-0.00236 R e .
\end{array}
\end{aligned}
$$

Eq. (7) is based on the experimental data of Castanet et al. [19] which were obtained for similar configurations of droplet streams and similar injection parameters than in the present study.

The resolution of the inverse problem begins with the simulation of the temporal evolution of the droplet diameter and temperature for several guess values of $\eta_{T}$ and $\eta_{M}$. To simplify the inversion, the values of $\eta_{T}$ and $\eta_{M}$ are maintained constant over the time period covered by the simulations. Then, the difference between the experimental data and the simulation is evaluated. Finally, the retained values of $\eta_{T}$ and $\eta_{M}$ are those that minimize the difference between the simulations and the experimental data. The accuracy of the adjustment is generally on the order of 0.1 for both $\eta_{T}$ and $\eta_{M}$ as illustrated in Fig. 9 in the case of ethanol droplets. It should be noted that an error on the estimate of $\eta_{M}$ is likely to affect $\eta_{T}$ and vice versa. As an example, it can be observed in Fig. 9 that taking $\eta_{M}=0.5$ (instead of 0.6 as normally given by the inverse method) leads to underestimate $\eta_{T}$ by also 0.1 . Furthermore, the reliability of the inverse method depends on the amplitudes of the size and temperature variations in the measurements. The sensitivity of the size and temperature evolutions to the parameter $\eta_{M}$ is poor for droplets of low-volatile liquids which do not evaporate significantly. Similarly, the sensitivity to $\eta_{T}$ is poor when the temperature is close to the equilibrium temperature for high-volatile fuels. In these two limit cases, only one parameter among $\eta_{T}$ and $\eta_{M}$ could be estimated by the inverse method. When both $\eta_{T}$ and $\eta_{M}$ could be determined, their estimates were generally found to be very close as illustrated in Fig. 9. This closeness is certainly due to the similitude between the heat and mass transfers, which can be rigorously established for unitary Lewis number.

\subsection{Summary of the estimations of Nusselt and Sherwood numbers from the experiments}

The present study is based on 57 test cases summarized in Table 1. It was possible to change the spacing parameter from about 2.5 to 10 in the experiments. Nevertheless, a regular breakup of the liquid jet into periodical droplets can be obtained for a more or less wide range of injection parameters depending on the properties of the injected liquid (viscosity, surface tension...). Also, it should be emphasized that the diameter of the droplets is not totally independent of the distance parameter. For a given injection pressure, the product $d^{2}$. $C^{-1}$ is proportional to the diameter of the injector orifice. Different fuels were tested including isohexane, ethanol, $n$-decane and $n$-dodecane. In the case of $n$-dodecane, several injection temperatures, corresponding to $T_{0}=20^{\circ} \mathrm{C}, 40^{\circ} \mathrm{C}$ $70^{\circ} \mathrm{C}$ and $170^{\circ} \mathrm{C}$, were tested. Except $n$-dodecane, no other fuel was preheated before entering the evaporation chamber.

In Fig. 10, the adjusted values of $\eta_{M, T}$ are plotted against the spacing parameter $C$ for the different fuels. Despite a significant scattering of data, some conclusions can be drawn from this figure. For a given fuel, $\eta_{M, T}$ increases with the spacing parameter, which is in line with the observations made in Section 3.2. For a given value of the spacing parameter, $\eta_{M, T}$ is generally lower for the less volatile droplets, namely the $n$-decane and $n$-dodecane droplets entering the chamber with $20^{\circ} \mathrm{C} \leqslant T_{0} \leqslant 70^{\circ} \mathrm{C}$. Hence, the spacing parameter seems to be not totally sufficient to describe the effects of the interactions on heat and mass transfers, which has been already noticed by Deprédurand et al. [7].

\section{Empirical data reduction}

The evaporation of a single droplet immersed in a hot air environment is dominated by coupled heat and mass transfers. If the droplet is motionless in a stagnant atmosphere, mass transport is mainly governed by diffusion, convection by the Stefan flow, and natural convection. Forced convection adds to the other transport phenomena in the presence of a relative velocity between the droplet and the gas. In the case of monodisperse droplet streams, thermal and concentration boundary layers for heat and mass transfer develop along the axis of the droplet streams and become thicker with the distance from the injector. Within these boundary layers, heat and mass transfers are mainly driven by the Stefan flow and diffusion. Convection by the Stefan flow and diffusion are eventually dominant compared to forced convection as the boundary layer thickness becomes much larger than the droplet radii. However, as it will be shown later in Section 5.5, the situation encountered at only a few centimeters from the injector is mixed, i.e. none of the transfer modes (forced convection, transport by the Stefan flow and diffusion) can be neglected.

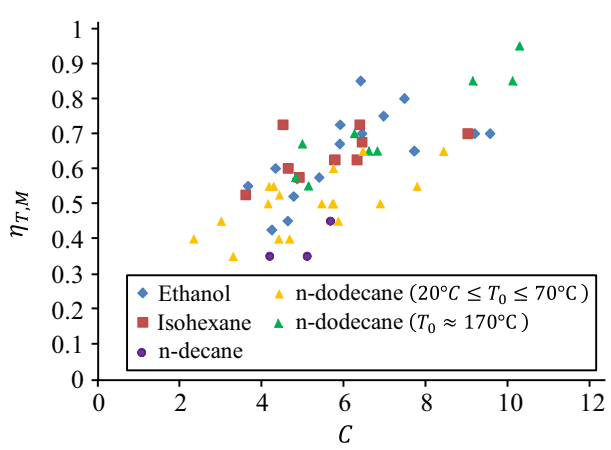

Fig. 10. The reduction coefficients $\eta_{M}$ and $\eta_{T}$ displayed as a function of the spacing parameter $C$ for all the tested experimental cases. $\eta_{M}$ and $\eta_{T}$ are determined from

\begin{tabular}{|c|c|c|c|c|c|c|}
\hline Fuel & $\begin{array}{l}\text { Boiling temperature } \\
{\left[{ }^{\circ} \mathrm{C}\right]}\end{array}$ & $\begin{array}{l}\text { Injection temperature } \\
{\left[{ }^{\circ} \mathrm{C}\right]}\end{array}$ & $C_{0}$ range & $\begin{array}{l}V_{0} \text { range } \\
{[\mathrm{m} / \mathrm{s}]}\end{array}$ & $\begin{array}{l}d_{0} \text { range } \\
{[\mu \mathrm{m}]}\end{array}$ & Number of test cases \\
\hline Isohexane & 60 & 30 & $3.6-9$ & $8.1-16.2$ & $83-128$ & 10 \\
\hline Ethanol & 78 & $30-50$ & $3.7-9.6$ & $9.3-14$ & $88-140$ & 17 \\
\hline \multirow[t]{4}{*}{ n-Dodecane } & 214 & 20 & $2.4-5.8$ & $8-13.5$ & $97-180$ & 9 \\
\hline & & 40 & $4.3-6.9$ & $8.5-13$ & $102-123$ & 4 \\
\hline & & 70 & $4.7-8.5$ & $9.2-13$ & $94-117$ & 5 \\
\hline & & 170 & $5-10.3$ & $13-15.3$ & $92-135$ & 9 \\
\hline$n$-Decane & 174 & 30 & $4.2-5.7$ & $12-13.7$ & $100-110$ & 3 \\
\hline
\end{tabular}
the experiments by solving the inverse problem described in Section 3.3.

Table 1

Summary of the injection conditions tested in the experiments. 


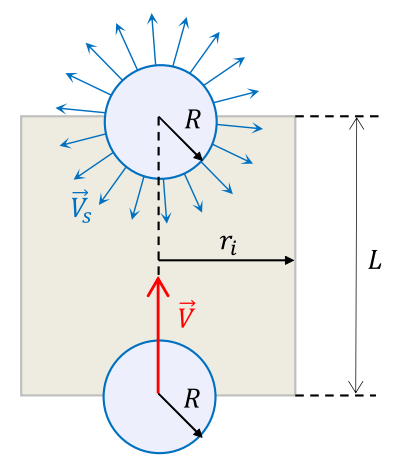

Fig. 11. The region of influence considered in the modeling in Section 4 .

As suggested by Imaoka and Sirignano [9], the influence of droplet interactions on heat and mass transfers in arrays of motionless droplets can be well described by the ratio $\xi=\vartheta_{g} / \vartheta_{l}$ between the gas volume $\vartheta_{g}$ and the liquid volume $\vartheta_{l}$ in the considered array. According to Sazhin et al. [20], the gas volume $\vartheta_{g}$ could be represented in the case of a single moving droplet by a region of influence surrounding the droplet inside of which heat and mass transfers between the gas and the droplet take place. This region is naturally set as a sphere in the case of an isolated droplet and as a cylinder when a chain of closely-spaced droplets is considered. In the following approach, the boundary layer that develops around the droplet chain is thus replaced by the region of influence. The diameter of this cylindrical region is related to the rate of the radial transport of vapor by the Stefan flow and diffusion, as well as the relative velocity between the droplet and the free stream (Fig. 11).

The radius of the cylinder of influence $r_{i}$ can be expressed by:

$r_{i}=R+V_{S} \cdot \tau+\sqrt{D \tau}$,

where $V_{s}$ is the Stefan flow velocity, $\tau$ is a characteristic time, $D$ is the diffusivity of heat or vapor. The characteristic height of the cylinder of influence is taken as the distance $L$ between two consecutive droplets, this choice being supported by the periodicity of the droplet row. Also because of the periodicity, the characteristic time $\tau$ is chosen as the time for a droplet to travel the distance $L$, i.e. $\tau=L / V$ where $V$ is the local droplet velocity. The radius of the cylinder of influence increases with the droplet spacing $L$ and decreases with the droplet velocity, which is obviously in favor of vapor and heat accumulation in the cylinder for the low distances $L$ between the droplets. For the high droplet velocities, the radius of the cylinder of influence is smaller. This also makes sense since the boundary layer associated with forced convection is of lesser thickness when the Reynolds number and thus the droplet velocity increase. According to Eq. (8), the volume of the cylinder of influence is given by:

$\vartheta_{g}=\pi r_{i}^{2} L-\frac{4}{3} \pi R^{3}$

The ratio $\xi=\vartheta_{g} / \vartheta_{l}$ can be thus calculated as:

$$
\begin{aligned}
\xi & =\frac{\vartheta_{g}}{\vartheta_{l}}=\left[\pi r_{i}^{2} L-\frac{4}{3} \pi R^{3}\right] / \frac{4}{3} \pi R^{3} \\
& =\left[\frac{3}{2} C+6 C^{3 / 2} \sqrt{v / 2}+3 C^{2} v+6 C^{2}\left((1+\sqrt{2 C v}) \kappa+C \kappa^{2}\right)\right]-1,
\end{aligned}
$$

where $C$ is the spacing parameter, $v=D /(V \cdot R)$ and $\kappa=V_{s} / V$ denotes the ratio between the velocity of the Stefan flow and the droplet velocity. The reduction coefficients $\eta_{M, T}$ are displayed as a function of the non-dimensional parameter $\xi$ in Fig. 12. Monodis-

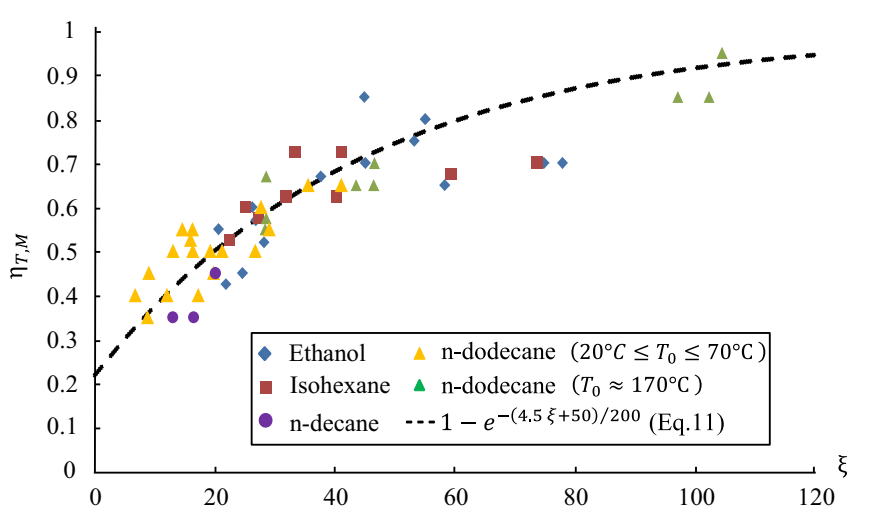

Fig. 12. Evolution of the reduction coefficients $\eta_{M}$ and $\eta_{T}$ as a function of the dimensionless parameter $\xi$ defined in Eq. (10).

perse droplet streams having low spacing parameters and low Stefan flow velocities (in practice low injection temperature and low volatility) correspond to the lowest values of $\xi$, while high Stefan flow velocities and high spacing parameter correspond to the highest values of $\xi$. Compared with Fig. 10, estimated values of $\eta_{M, T}$ seem to be better correlated with the parameter $\xi$ than the spacing parameter $C$. This can be certainly explained by the fact that $\xi$ includes at the same time the effects of the droplet spacing and the Stefan flow. Data scattering in Fig. 12 is compatible with the accuracy of the adjustment of $\eta_{M, T}$ (on the order of \pm 0.1 ). Some effects (like the effect of the Reynolds number, and the distance from the injector) are probably missing or not well accounted for, as they are more difficult to extract from the other parameters with the current set of experimental results. A correlation for $\eta_{M, T}$ based on the parameter $\xi$ can be proposed:

$\eta_{M, T} \approx 1-\exp \left(-\frac{4.5 \xi+50}{200}\right)$, with $0 \leqslant \xi \leqslant 120$.

The main trend observed in Fig. 12 is well captured by this expression. The differences between Eq. (11) and the values of $\eta_{M, T}$ determined experimentally are generally less than 0.1 which roughly corresponds to the uncertainties on the estimate of $\eta_{M, T}$ using the inverse method described in Section 3.3.

\section{Numerical study of the droplet interactions}

Numerical simulation is also relevant in order to study the interaction between neighbor drops. However, the limitation of available computing resources is a major constraint which implies making some simplifications. In the early studies on droplet arrays, forced and natural convection were usually not accounted for $[21,22]$. Convection was limited to the Stefan flow. The simplified problem can be resolved very efficiently using mass-flux potential functions which are solutions to a Laplace's equation in the gas field. The method is very efficient to handle a large number of droplets in the arrangement. In a recent study, up to 216 drops arranged three-dimensionally were considered by Imoka and Sirignano $[9,23]$. In comparison, arrangements remain limited to much fewer droplets in the numerical studies where the transient resolution of Navier-Stokes, energy and species equations are solved to account for the convective evaporation of interacting droplets. The early work of Chiang and Sirignano [10] considers only two or three droplets moving in tandem. However, many physical features are included in the computations such as droplet regression, deceleration of the stream flow, liquid motion, temperaturedependent properties, ... Recent formulation of the problem with periodical boundary conditions allowed extending the simulations 
to infinite periodic arrays with one or more rows of droplets $[11,12,24]$. The case of an infinite array with a single row is rather comparable to our experiments, even though real droplet streams are semi-infinite.

\subsection{Formulation of the problem}

Given the large number of droplets to consider for a comparison with the experiments, a simplified approach has been developed. It does not incorporate all the phenomena, but still considers key physical features like the forced convection in the array, the formation of boundary layers around the axis of the droplet row. The assumptions on which this approach is based are summarized in the following:

1. The problem is assumed to be stationary. Hence, the droplet size does not change despite the evaporation and the thermal expansion of the liquid. Also the deceleration of the droplet due to the drag is not considered. This implies that the distance between the droplets is not changing with time.

2. The temperature is constant inside the droplet to satisfy 1 ), but it is also uniform over the droplet surface. Consequently, due to a liquid/vapor equilibrium, the vapor concentration is also constant and uniform at the droplet surface.

3. The droplets are rigid spheres. There is zero tangential velocity at the liquid surface. Only the normal velocity, corresponding to the Stefan flow, is considered.

4. The physical properties are uniform. They do not depend on the composition and temperature in the gas flow. In particular, the diffusion coefficient for vapor is a constant.

These assumptions are classically invoked to derive the $d^{2}$-law in the case of the isolated droplet $[17,18]$. As the temperature and the vapor mass fraction are imposed at the droplet surface, the problem is reduced to the transport of heat, mass and momentum in the gas phase. The steady continuity equation, NavierStokes equations, energy and species conservation equations apply:

$\nabla \cdot \vec{u}=0$

$\rho(\vec{u} \cdot \nabla) \vec{u}=\nabla \cdot\left[-p I+\mu\left(\nabla \vec{u}+(\nabla \vec{u})^{T}\right)\right]$

$\nabla \cdot\left(-D_{v} \nabla Y\right)+\vec{u} \cdot \nabla Y=0$

$\nabla \cdot\left(-\lambda_{g} \nabla T\right)+\rho_{g} C p_{g} \vec{u} \cdot \nabla T=0$.

At the surface of the droplet, the coupling between the transports of heat and mass can be expressed as:

$\vec{V}_{s}=\dot{m}^{\prime \prime} / \rho_{g} \vec{n}$,

$\dot{m}^{\prime \prime}\left(1-Y_{s}\right)=-\left.\rho_{g} D_{v} \frac{\partial Y}{\partial n}\right|_{s}$,

$\left.\lambda_{g} \frac{\partial T}{\partial n}\right|_{s}=L v_{e} \dot{m}^{\prime \prime} \quad$ with $\quad L v_{e}=L v+Q_{L}^{\prime \prime} / \dot{m}^{\prime \prime}$

The terms $Q_{L}^{\prime \prime}$ and $\dot{m}^{\prime \prime}$ correspond to the heat flux entering the droplet, and the vapor mass flow rate per unit area. The unit normal vector to the drop surface is denoted by $\vec{n}$ and $\vec{V}_{s}$ denotes the velocity of the Stefan flow. Without loss of generality, the resolution can be restricted to the mass transfer. The problem formulated by Eqs. (12)-(14), (16) and (17) is applied to the case of a line of periodically-arranged droplets. The computational domain is illustrated in Fig. 13. The problem can be solved in the cylindrical coordinates $(r, z)$ given the axisymmetry of the flow configuration. Using the indexing numbers in Fig. 13, the boundary conditions can be specified as follows:

- Boundary 1: Inlet

$$
\begin{aligned}
& \vec{u}=u_{d} \vec{n}, \\
& Y=Y_{\infty} .
\end{aligned}
$$

Given that the droplets are fixed in the reference frame of the numerical study, the gas velocity $u_{d}$ is the opposite of the droplet velocity $V$ in the laboratory frame of reference.

- Boundary 2: Axial symmetry

$$
\frac{\partial Y}{\partial r}=0, \quad \frac{\partial u_{r}}{\partial r}=0, \quad \frac{\partial u_{z}}{\partial r}=0 .
$$

- Boundary 3: Open boundary

$$
\begin{gathered}
\left\{\begin{array}{l}
-\vec{n} \cdot D_{v} \vec{\nabla} Y=0 \text { if } \vec{n} \cdot \vec{u} \geqslant 0 \\
Y=Y_{\infty} \text { if } \vec{n} \cdot \vec{u}<0
\end{array},\right. \\
{\left[-p \mathrm{I}+\mu\left(\nabla \vec{u}+(\nabla \vec{u})^{T}\right)\right] \vec{n}=\overrightarrow{0} .}
\end{gathered}
$$

- Boundary 4: Droplet surface

$$
\begin{aligned}
& Y=Y_{s}, \\
& \vec{u}=V_{s} \vec{n} \quad \text { with } \quad V_{s}=-\left.\frac{D_{v}}{\left(1-Y_{s}\right)} \frac{\partial Y}{\partial n}\right|_{s} .
\end{aligned}
$$

\subsection{Solution procedure}

Eqs. (12)-(14) supplemented with the boundary conditions (19)-(25) are solved using the finite element method relying on an unstructured triangular mesh of increased density near the droplet surface (typically the half-perimeter of the droplets is divided into 100 elements of equal size). One difficulty for the resolution is to satisfy conditions (24) and (25) at the same time. The coupling between the flow velocity and the mass flux in Eq. (25) requires implementing an iterative procedure:

- 1 st iteration: The velocity field, denoted $u^{1}$ at the 1 st iteration, is solved based on Eq. (12) and Eq. (13) assuming a zero velocity of the Stefan flow $\left(V_{s}^{1}=0\right)$. The obtained velocity field $u^{1}$ is then injected in Eq. (14) to determine the distribution of the vapor mass fraction $Y^{1}$.

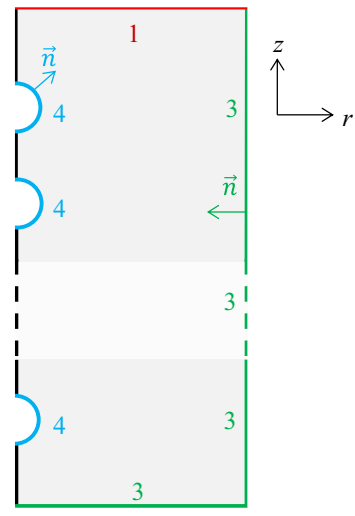

Fig. 13. Illustration of the computational domain and specification of the boundary conditions considered in the simulations. 
- 2nd iteration: Using $Y^{1}$, the Stefan flow can be approximated with $V_{s}^{2}=-D_{v} /\left(1-Y_{s}^{1}\right) \partial Y^{1} / \partial n$. The velocity $u_{s}^{2}$ is then considered in Eq. (25) for the resolution of the gas flow. The updated velocity field $u^{2}$ is injected in the vapor transport equation to determine the field of vapor mass fraction $Y^{2}$.

- ith iteration: After the 2nd iteration, the velocity of the Stefan flow is calculated using the following expression:

$$
V_{s}^{i}=\frac{1}{2}\left(V_{s}^{i-1}-\frac{D_{v}}{1-Y_{s}^{i-1}} \frac{\partial Y^{i-1}}{\partial n}\right) \text {. }
$$

This numerical scheme allows the convergence of the calculations when the velocity of the Stefan flow is non-negligible compared with that in the free stream $u_{d}$. In particular, this occurs for the high $B_{M}$ (typically $B_{M}>2$ ). For each droplet, the spatially-averaged Sherwood number is evaluated using:

$$
\langle S h\rangle_{j}=\frac{2}{\pi R^{2}} \int_{z_{j}-R}^{z_{j}+R} \operatorname{Sh}(r, z) r d z \text { with } r=\sqrt{R^{2}-z^{2}} .
$$

In this expression, $z_{j}$ denotes the axial position of the $j$ th droplet in the row. $R$ is the droplet's radius and $\operatorname{Sh}(r, z)$ corresponds to the local Sherwood number defined by:

$\operatorname{Sh}(r, z)=-\left.\frac{d}{\left(Y_{s}-Y_{\infty}\right)} \frac{\partial Y}{\partial n}\right|_{s}$

The iterative calculation is stopped when the variations of the Sherwood number $\langle S h\rangle$ between two consecutive iterations become very small:

$\sup _{j}\left(\frac{\left|\langle S h\rangle_{j}^{i}-\langle S h\rangle_{j}^{i-1}\right|}{\langle S h\rangle_{j}^{i}+\langle S h\rangle_{j}^{i-1}}\right) \leqslant \frac{\varepsilon}{2}$.
In the results presented thereafter, the value of $\varepsilon$ was fixed at $10^{-4}$. About 8 iterations are usually sufficient to satisfy Eq. (28). The case $B_{M}=0$ is a special one, since the velocity of the Stefan flow is null. When $B_{M}=0$, calculations are stopped after only one iteration and $Y_{S}$ is set to an arbitrary value different from $Y_{\infty}$ in order to determine the Sherwood number using Eq. (27).

\subsection{Numerical validation against the isolated droplet}

The previously described numerical approach was validated against existing models relative to the isolated droplet. The wellknown correlation of Ranz and Marshall [25] which applies to non-evaporating droplet, can be used for the evaluation of $N u_{0}$ and $S h_{0}$ in the absence of Stefan flow $\left(B_{M}=0\right)$ :

$$
\begin{aligned}
& N u_{0}=2+0.6 \operatorname{Re}^{1 / 2} \operatorname{Pr}^{1 / 3}, \\
& S h_{0}=2+0.6 \operatorname{Re}^{1 / 2} S c^{1 / 3}
\end{aligned}
$$

An alternative, more recommended than Eq. (29) at low Reynolds numbers (typically $R e \leqslant 10$ ), is the correlation of Clift et al. [26]:

$$
\begin{aligned}
& N u_{0}=1+(1+\operatorname{RePr})^{1 / 3} \cdot f(R e), \\
& S h_{0}=1+(1+\operatorname{ReSc})^{1 / 3} \cdot f(\operatorname{Re}),
\end{aligned}
$$

where $f(R e)=1$ at $R e \leqslant 1$ and $f(R e)=R e^{0.077}$ at $R e \leqslant 400$. The deviation between the simulations and Eq. (30) is displayed in Fig. 14a. In the worst case, differences do not exceed $3.5 \%$ in the range $0.2 \leqslant S c \leqslant 4$ and $2 \leqslant R e \leqslant 100$. For the evaporative case $\left(B_{M}>0\right)$, numerical results can be compared with the film theory [8]:

$$
\begin{aligned}
& N u_{\text {iso }}=\frac{\ln \left(1+B_{T}\right)}{B_{T}}\left[2+\frac{\left(N u_{0}-2\right)}{F_{T}}\right], \\
& S h_{\text {iso }}=\frac{\ln \left(1+B_{M}\right)}{B_{M}}\left[2+\frac{\left(S h_{0}-2\right)}{F_{M}}\right],
\end{aligned}
$$
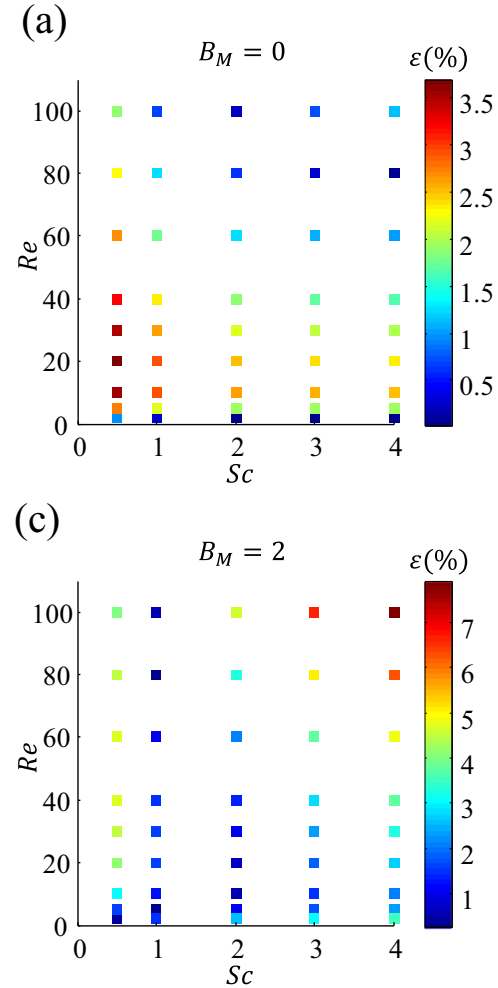
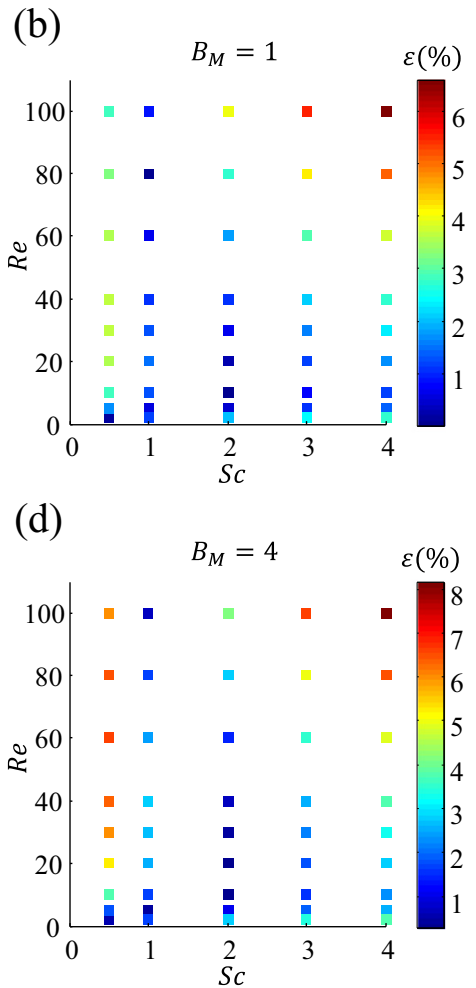

Fig. 14. Relative differences in percent between the Sherwood numbers calculated with the current numerical approach applied to an isolated droplet and Eq. (31) associated Eq. (30). 
where $F_{M}=F\left(B_{M}\right), F_{T}=F\left(B_{T}\right)$ and $F=(1+B)^{0.7} \frac{\ln (1+B)}{B}$. As shown in Fig. 14(b-d), differences between the simulations and Eq. (31), with the use of Eq. (30) for the evaluation of $S h_{0}$, do not exceed $8 \%$ in the range $0.2 \leqslant S c \leqslant 4,2 \leqslant R e \leqslant 100$ and $0 \leqslant B_{M} \leqslant 4$. However, a general trend is an increase of the discrepancies with increasing Spalding number $B_{M}$.

\subsection{Heat and mass transfer distributions in a row of interacting droplets}

In the following, single rows of 80 equally-spaced droplets are considered in the simulations. About 800 simulations were carried out to cover the range: $10 \leqslant R e \leqslant 100,0.5 \leqslant S c \leqslant 4,1.1 \leqslant C \leqslant 18$ and $0 \leqslant B_{M} \leqslant 4$. Fig. 15 shows the distributions of velocity and vapor mass fraction around the droplets in the case $C=4, R e=40, B_{M}=2$ and $S c=1$. The gas velocity and the vapor mass fraction are affected by the presence of the droplets in a layer of gas, whose thickness increases progressively with the distance to the leading droplet. The evolutions of this layer thickness are comparable to the development of a boundary layer at the surface of a cylinder in a coaxial flow. Gradients of vapor concentration and velocity are not distributed uniformly at the surface of the droplets. The transfers are more intense near the leading edge of the droplets. As displayed in Fig. 16, the Sherwood number takes significantly higher values for angles of attack under $90^{\circ}$. Due to the closeness of the droplets, the maximum of the Sherwood number is not situated at an angle of attack equal to $0^{\circ}$ (except for the head droplet) but rather close to $50^{\circ}$. Near the trailing edge, the Sherwood number becomes significantly smaller than $2 \ln \left(1+B_{M}\right) / B_{M} \approx 1.1$, which can be certainly explained by the accumulation of vapor transported from the upstream. For sufficiently large Re, the presence of vortices in the wake of the droplets also promotes the vapor enrichment in the region of the trailing edge. In Fig. 16, the Sherwood number decreases with the distance to the leading droplet

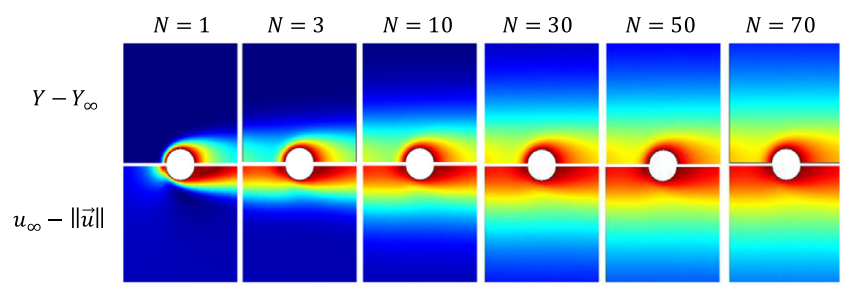

Fig. 15. Distribution of the vapor mass fraction and gas velocity in a single row of 80 drops. $N$ denotes the number of the droplet within the row of droplets. The simulation corresponds to $R e=40, C=4, B_{M}=2$ and $S c=1$.

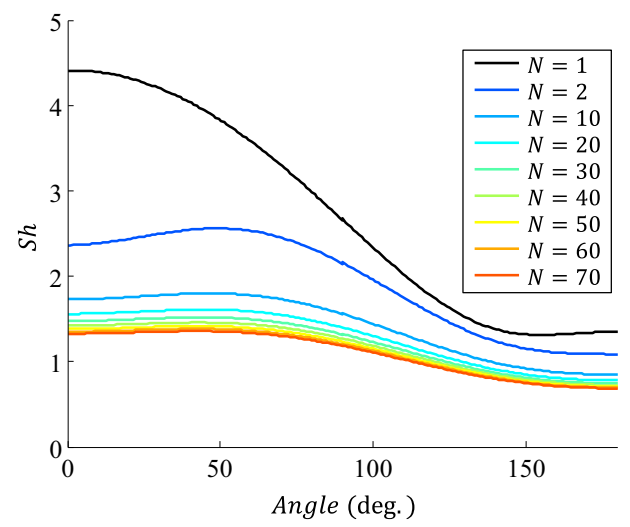

Fig. 16. Angular distribution of the Sherwood number at the droplet surface in the same conditions as Fig. 15.

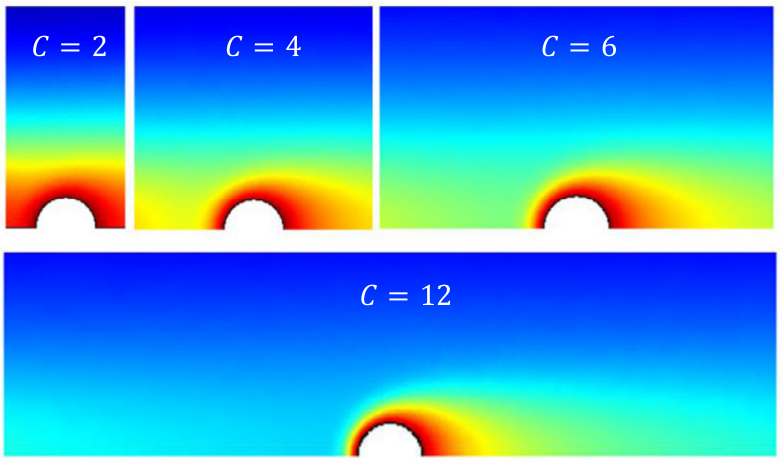

Fig. 17. Distribution of the vapor mass fraction around the 40th droplets for different values of the spacing parameter and $R e=40, B_{M}=2$ and $S c=1$.

and thus the droplet number $N$ in the row ordered from the leading droplet. Beyond $N=20$, the decrease of $S h$ with the droplet number $N$ becomes hardly visible, but strictly speaking no asymptotic limit can be invoked for $S h$, as the laminar boundary layer surrounding the droplet line keeps on developing with the axial distance $z$.

Still for $R e=40, B_{M}=2$ and $S c=1$, the distribution of the vapor mass fraction around the droplets was determined for different values of the spacing parameter. The images presented in Fig. 17 relate to the 40th droplets within the row and show the vapor distribution over a length $L$ equal to the inter-droplet distance $C \cdot d$. As expected, the wake effects are much more important for the small spacing parameters. For $C=2$, differences between the leading edge and the trailing edge can be hardly pointed out in Fig. 17. A more refined analysis in Fig. 18 shows that the mass gradient at the droplet surface and thus the Sherwood number are maximum at an angle of attack of about $70^{\circ}$. In fact, as $C$ tends to 1 , the maximum of the Sherwood number shifts toward an angle of $90^{\circ}$ and the difference between the leading and the trailing edge vanishes. In Fig. 18, a marked increase of Sh with $C$ can be observed. However, even for $C=12$, the distribution of the vapor mass fraction remains significantly influenced by the neighbor drops (Fig. 17) and the Sherwood number is far from having reached the values of the isolated droplet. A good approximation for $S h_{\text {iso }}$ is given by the Sherwood number of the leading droplet displayed in Fig. 16.

\subsection{Correlation for heat and mass transfers}

Based on the numerical simulations, a correlation can be determined for the Nusselt and Sherwood numbers of the interacting droplets. For the averaged Sherwood number, the following expression is proposed:

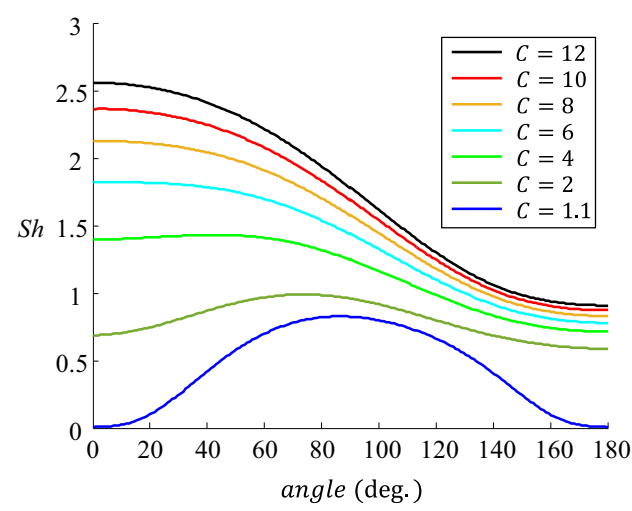

Fig. 18. Angular distribution of the Sherwood number at the surface of the 40th droplet. Parameters of the calculations are the same as in Fig. 17. 
$S h=\frac{\ln \left(1+B_{M}\right)}{B_{M}}\left[\kappa+\alpha \cdot\left(\frac{z}{d}\right)^{\beta} \frac{R e^{0.58+\gamma} S c^{\delta}}{F_{M}}\right]$

where $F_{M}=\left(1+B_{M}\right)^{0.655} \ln \left(1+B_{M}\right) / B_{M}$ and $z$ denotes the distance from the leading droplet in the row. The same expression can be used to evaluate the Nusselt number replacing $B_{M}$ by $B_{T}$ and $S c$ by $\operatorname{Pr}$. The formulation adopted in Eq. (32) has some similarities with the Ranz-Marshall's correlation (29) corrected from the effect of the Stefan flow by the film theory using Eq. (31). The isolated droplet can be obtained by setting $\alpha=0.6, \beta=0, \gamma=-0.08, \delta=1 / 3$ and $\kappa=2$. The only difference concerns the exponent 0.655 in the expression $F_{M}$ instead of 0.7 in Eq. (31). Using this exponent was found to improve substantially the quality of the estimations of the unknown parameters in Eq. (32). Parameters $\alpha, \beta, \gamma, \delta$ and $\kappa$ in Eq. (32) were estimated from about 90 simulations for each of the tested spacing parameters. The results summarized in Table 2 are valid for $10 \leqslant R e \leqslant 100,0.5 \leqslant S c \leqslant 4,0 \leqslant B_{M} \leqslant 4$ and $3 \leqslant z /(C \cdot d) \leqslant 76$. The errors indicated in the last column of Table 2 correspond to the differences between the average Sh calculated in the simulations and by Eq. (32). Globally, the errors are of the order of $1 \%$ for all the studied spacing parameters. For large spacing parameters, it can be verified in Fig. 19 that $\alpha, \beta, \gamma$ and $\kappa$ tend asymptotically to their prescribed value in the case of the isolated droplet. One noticeable exception is for $\delta$ which remains close to 0.5 . Far from the leading droplet, convective effects become insignificant due to the negative sign of $\beta$. This is a consequence of the development of the boundary layer around the row of droplets. The thickness of the boundary layer increases with $z$ and eventually becomes very large compared with the droplet size. For $z \gg d$, the problem comes down to motionless droplets and

Table 2

Estimated values of the parameters $\alpha, \beta, \gamma, \delta$ and $\kappa$ in Eq. (32) based on numerical simulation.

\begin{tabular}{llllll}
\hline$C$ & $\alpha$ & $\beta=\gamma$ & $\kappa$ & $\delta$ & $\operatorname{Err}(\%)$ \\
\hline 1.1 & 0.78063 & -0.28212 & 0.37587 & 0.29201 & 1.415 \\
2 & 1.0868 & -0.2733 & 0.67671 & 0.29955 & 1.322 \\
4 & 1.1403 & -0.23348 & 1.0596 & 0.34237 & 1.155 \\
6 & 1.1059 & -0.2034 & 1.2553 & 0.37656 & 1.032 \\
8 & 1.0337 & -0.17815 & 1.3788 & 0.40169 & 1.095 \\
10 & 0.96535 & -0.15754 & 1.4588 & 0.42261 & 1.165 \\
12 & 0.92053 & -0.14103 & 1.5038 & 0.43539 & 1.150 \\
15 & 0.8561 & -0.12179 & 1.5753 & 0.44429 & 1.085 \\
18 & 0.7846 & -0.10691 & 1.6491 & 0.47262 & 1.057 \\
\hline
\end{tabular}

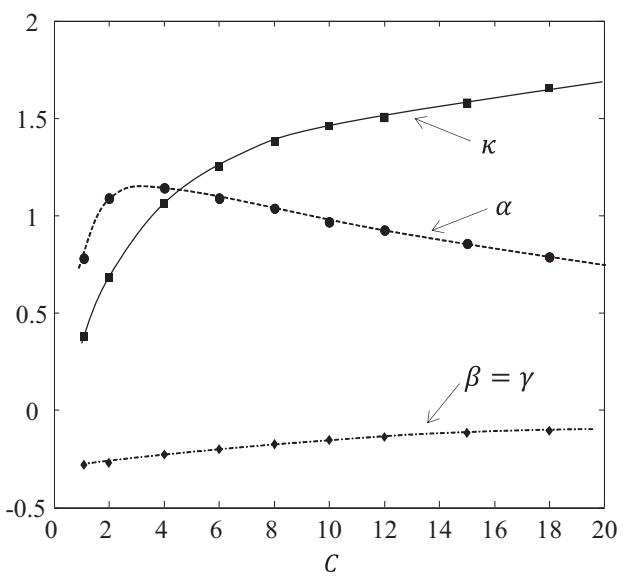

Fig. 19. Evolution of the adjusted parameters in Eq. (32). the Sherwood number can be simplified as $\kappa(C) \cdot \ln \left(1+B_{M}\right) / B_{M}$. Choosing for $z$ the distance covered by the droplets inside the chamber (typically a few centimeters), the term $(z / d)^{\beta}$ is found to be on the order of 0.4-0.7 in the conditions of the experiments. Hence, the convective term in Eq. (32) decreases slowly with $z$ due to the thickening of the boundary layer. Given the values of Re (typically a few tenths), convection still plays an important role in the experimental results, although being significantly reduced compared to the isolated droplet.

\section{Applications of the model and overall validations}

In this section, the empirical correlation (11) and the correlation (32) derived from the numerical simulations are compared with the experimental results for validation. In a first stage, Nusselt and Sherwood numbers are determined using Eq. (32) for all the experimental cases tested in the study. For the calculations, averaged values of $R e, B_{M}, B_{T}, P r$ and $S c$ given by the resolution of the inverse problem in Section 3.4 are taken in Eq. (32), while the distance $z$ to the chamber entrance is fixed to $1.5 \mathrm{~cm}$. Nusselt and Sherwood numbers are then divided by $\mathrm{Nu}_{\text {iso }}$ and $S h_{\text {iso }}$ using Eqs. (30) and (31), which allows evaluating the reduction coefficients $\eta_{M . T}$. In Fig. 20, these values of $\eta_{M . T}$ derived from Eq. (32) are plotted against the coefficients $\eta_{M, T}$ estimated from the measurements solving the inverse problem described in Section 3. It is observed that the numerical correlation (32) is able to reproduce quite well the experimental results for a large number of cases. The differences between the experimental results and the numerical model are relatively small for most cases, i.e. they fall with the uncertainty margin of \pm 0.1 corresponding to the estimate of $\eta_{M . T}$ by the inverse method presented in Section 3.3. Nevertheless, for a dozen of cases, the values of $\eta_{M, T}$ given by the numerical correlation (32) are noticeably higher than their experimental counterparts. For these cases, there is an additional gap of about 0.1 between the experiments and the numerical correlation, which seems difficult to explain.

Finally the experimental correlation (11) and the numerical correlation (32) are used to simulate the size and temperature evolutions of the droplets. These correlations are incorporated into the direct model upon which the resolution of the inverse problem described in Section 3.3 is based. The results of these simulations are compared with the experimental data in Figs. 21-24 for a limited number of cases among the 55 investigated experimentally. Some trends emerge from the comparisons:

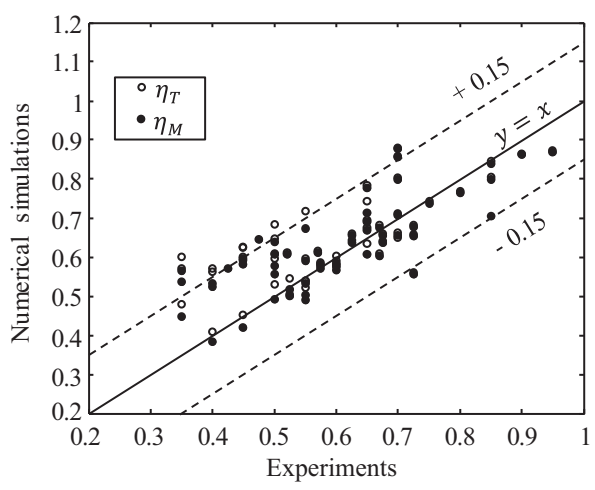

Fig. 20. Estimations of the reductions coefficient $\eta_{M T}$. The values of $\eta_{M, T}$ derived from Eq. (32) are plotted against their counterparts obtained from the experiments using the inverse method in Section 3.3. 


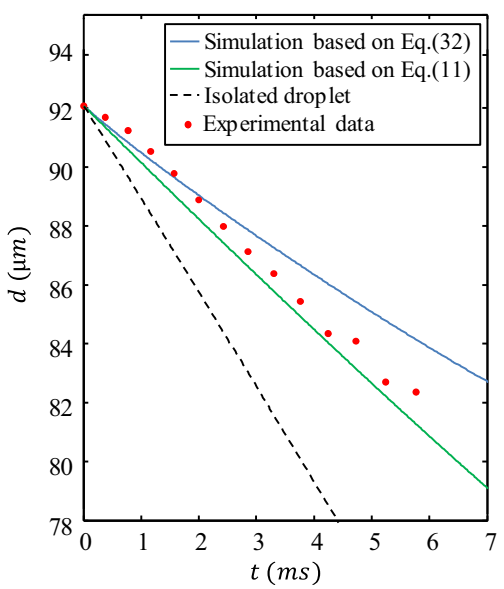

Fig. 21. Time evolution of the size for $n$-dodecane droplets evaporating at their equilibrium temperature $\left(C_{0}=4.8, d_{0}=92 \mu \mathrm{m}, V_{0}=13.2 \mathrm{~m} / \mathrm{s}, T_{0}=170{ }^{\circ} \mathrm{C}\right)$. Comparisons between measurements and simulations based on Eqs. (11) and (32).

- The largest deviations between the numerical correlation (32) and the experiments were observed for the $n$-dodecane droplets evaporating at the equilibrium temperature. As shown in
Fig. 21, the experimental correlation (11) agrees well with the size measurements while the numerical correlation (32) systematically underestimates the evaporation rate of the droplets.

- In the case of ethanol, the two correlations allow obtaining satisfying agreements with the measurements. However, the quality of the predictions varies from one case to another. In other words, one of the two correlations can match the experimental data while the other not, and vice versa. For example, the numerical simulation based on Eq. (32) gives better results in Fig. 22, but the contrary is observed in Fig. 23. Given that the experimental conditions are rather similar for the cases presented in these figures, a certain variance is probably to be considered among the experimental results. The same variance has been already pointed out in Figs. 10 and 12. Fig. 23 relates to the same experimental case as presented in Fig. 9. Based on this figure, it can be verified that the values of $\eta_{M}$ and $\eta_{T}$ predicted by the numerical correlation (32) are close to 0.5 , that is about 0.1 below the estimates from the inverse method.

- For the less volatile fuels, namely $n$-decane and $n$-dodecane (at $T_{0} \leqslant 70^{\circ} \mathrm{C}$ ), both correlations were found to generally give acceptable results as illustrated in Fig. 24

- It can be observed in Figs. 21-24 that the numerical correlation (32) always underestimates the heating and evaporation rates compared to the experimental correlation (11) based on the $\xi$ (a)

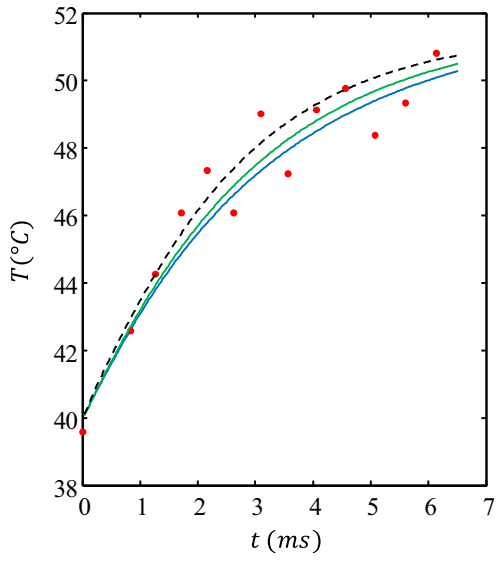

(b)

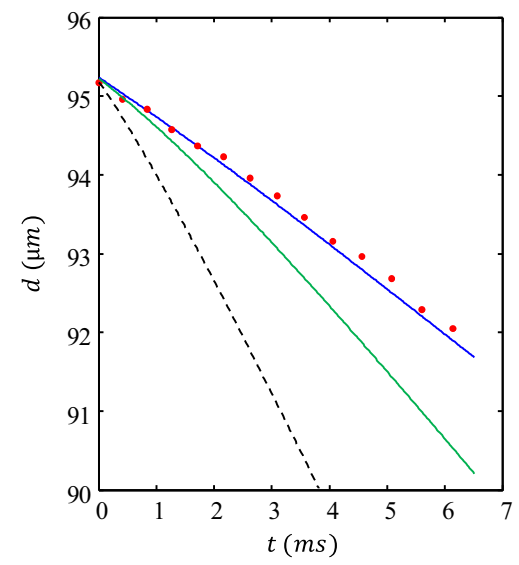

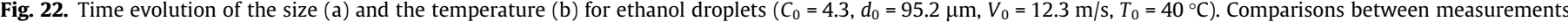
and simulations based on Eqs. (11) and (32). The legend is the same as in Fig. 21.

(a)

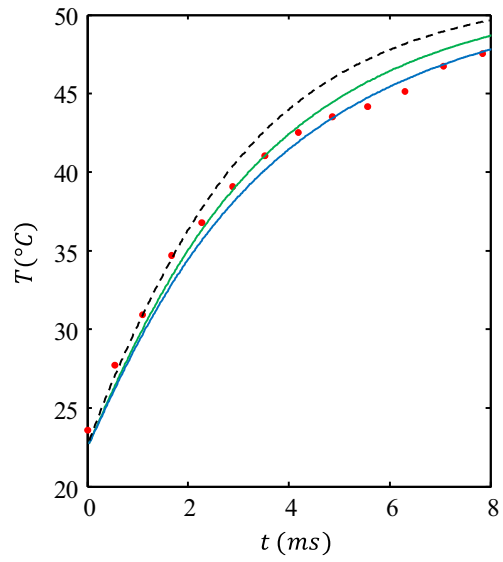

(b)

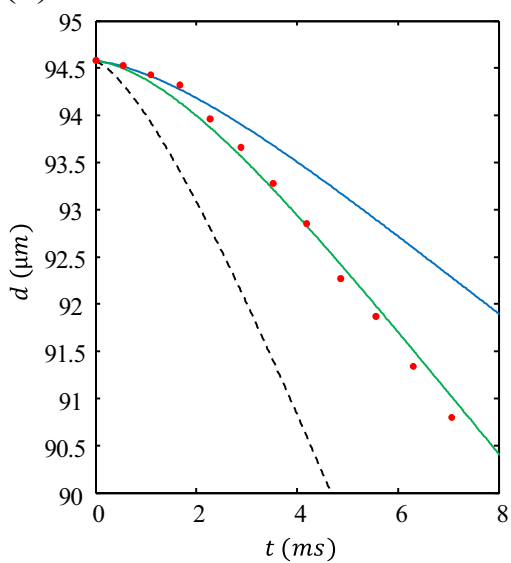

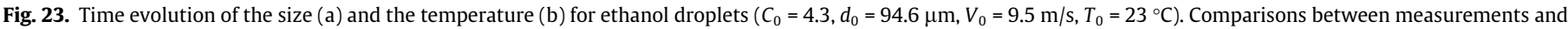
simulations based on Eqs. (11) and (32). The legend is the same as in Fig. 21. 
(a)

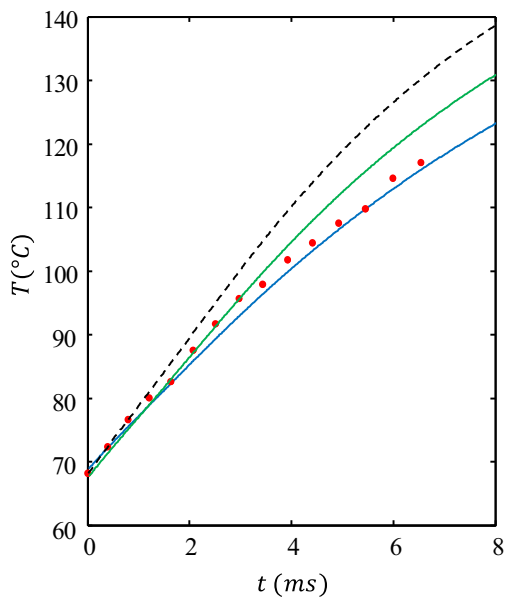

(b)

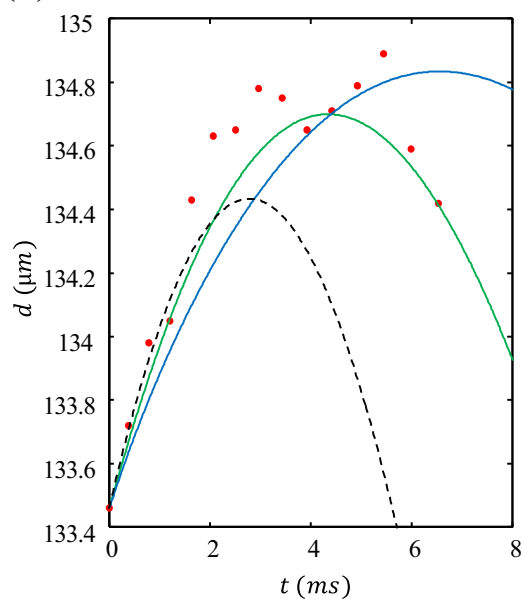

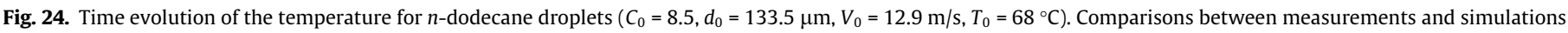
based on Eqs. (11) and (32). The legend is the same as in Fig. 21.

parameter. It should be emphasized that any perturbation of the boundary layer in the experiments is likely to increase the intensity of the heat and mass transfers in comparison with the ideal laminar case considered in the simulations carried out in Section 5. These perturbations may originate from the air co-flow injected into the chamber to prevent vapor accumulation. Furthermore, the droplet streams eventually loses its periodicity after a few centimeters, some droplets being slowed down more rapidly than their neighbors. Therefore, it is probably acceptable to obtain with the numerical simulations some values of $\eta_{M}$ and $\eta_{T}$ that are generally slightly lower than in the experiments.

\section{Conclusions}

The heating and evaporation of interacting droplets are studied experimentally using lines of periodically-arranged mono-sized droplets injected in a chamber where a high ambient gas temperature is imposed. Advanced optical techniques are applied to characterize the evolution of the droplet size and temperature for several fuels and different injection conditions. Characteristic heat and mass transfer numbers, namely the Sherwood and Nusselt numbers, are estimated from the experimental results and compared to the classical model of the isolated droplet in order to estimate the effects of droplet interactions on the rate of heat and mass transfers. The droplet spacing appears to have a strong influence on the reduction of the Nusselt and Sherwood numbers, but this parameter alone fails to explain the variations of the Nusselt and Sherwood numbers observed in the set of experimental results.

In a first approach, an empirical correlation is derived from the experimental results. Based on the concept of volume of influence, a single dimensionless parameter is introduced to account for the transport of heat and vapor by the Stefan flow and the diffusion in a region of influence that mimics the boundary layer that develops around the chain of droplets. As the thickness of the boundary layer surrounding the chain of droplets increases with the distance from the injector, forced convection has a more and more limited influence on the heat and mass transport. Inside the boundary layer, the transfers are mainly governed by diffusion and convection by the Stefan flow. The radius of the volume of influence depends on the rate of the radial transport of vapor by the Stefan flow and diffusion, as well as the relative velocity between the droplet and the free stream.

In a second approach, numerical simulations based on the resolution of the Navier-Stokes equations and the vapor transport equation are carried out to evaluate the Nusselt and Sherwood numbers of droplets arranged in single rows of 80 periodicallyspaced droplets. To simplify the numerical resolution, it is assumed that the problem is stationary and the droplets are assimilated to rigid spheres. Beside these approximations, key physical features for the understanding of the interaction effects are taken into account like the forced convection in the droplet array, the development of a boundary layer along the axis of the droplet row. A parametric study of the influence of the main characteristic numbers involved in the transfers allows to infer a correlation for Nusselt and Sherwood numbers that is valid for $10 \leqslant R e \leqslant 100,0.5 \leqslant$ $S c \leqslant 4,0 \leqslant B_{M} \leqslant 4$ and $3 \leqslant z /(C \cdot d) \leqslant 76$.

Finally, both the empirical and the numerical correlations established previously are shown to give acceptable results when their predictions are compared to the experiments. These new correlations are a step forward to the modeling of the evaporation of interacting droplets, since they include the effect of the droplet spacing, the influence of the fuel volatility, the configuration of the flow which gives rise to a boundary layer developing along the droplet chain in the case of one-dimensional rows of droplets.

\section{Acknowledgment}

The authors acknowledge the French Agence Nationale de la Recherche for its financial support of through the Grant No. ANR11-ASTR-0023 (DEVACOL).

\section{References}

[1] M. Labowsky, Calculation of the burning rates of interacting fuel droplets, Combust. Sci. Technol. 22 (5-6) (1980) 217-226, http://dx.doi.org/10.1080/ 00102208008952385.

[2] J. Sangiovanni, M. Labowsky, Burning times of linear fuel droplet arrays: a comparison of experiment and theory, Combust. Flame 47 (1982) 15-30, http://dx.doi.org/10.1016/0010-2180(82)90086-4.

[3] M. Marberry, A. Ray, K. Leung, Effect of multiple particle interactions on burning droplets, Combust. Flame 57 (3) (1984) 237-245, http://dx.doi.org/ 10.1016/0010-2180(84)90043-9.

[4] J. Virepinte, Y. Biscos, G. Lavergne, P. Magre, G. Collin, A rectilinear droplet stream in combustion: droplet and gas phase properties, Combust. Sci. Technol. $150 \quad(1-6) \quad(2000) \quad 143-159$, http://dx.doi.org/10.1080/ 00102200008952121. 
[5] G. Castanet, M. Lebouché, F. Lemoine, Heat and mass transfer of combusting monodisperse droplets in a linear stream, Int. J. Heat Mass Transfer 48(16)(2005) 3261-3275, http://dx.doi.org/10.1016/j.ijheatmasstransfer.2005.03.005.

[6] G. Castanet, P. Lavieille, F. Lemoine, M. Lebouché, A. Atthasit, Y. Biscos, G. Lavergne, Energetic budget on an evaporating monodisperse droplet stream using combined optical methods: evaluation of the convective heat transfer, Int. J. Heat Mass Transfer 45 (25) (2002) 5053-5067, http://dx.doi.org/10.1016/ S0017-9310(02)00204-1.

[7] V. Deprédurand, G. Castanet, F. Lemoine, Heat and mass transfer in evaporating droplets in interaction: influence of the fuel, Int. J. Heat Mass Transfer 53 (1718) (2010) 3495-3502, http://dx.doi.org/10.1016/j.ijheatmasstransfer.2010.04.010.

[8] B. Abramzon, W. Sirignano, Droplet vaporization model for spray combustion calculations, Int. J. Heat Mass Transfer 32 (9) (1989) 1605-1618, http://dx.doi. org/10.1016/0017-9310(89)90043-4.

[9] R.T. Imaoka, W.A. Sirignano, Transient vaporization and burning in dense droplet arrays, Int. J. Heat Mass Transfer 48 (2122) (2005) 4354-4366, http:// dx.doi.org/10.1016/j.ijheatmasstransfer.2005.05.012.

[10] C. Chiang, W. Sirignano, Interacting, convecting, vaporizing fuel droplets with variable properties, Int. J. Heat Mass Transfer 36 (4) (1993) 875-886, http://dx. doi.org/10.1016/S0017-9310(05)80271-6.

[11] G. Wu, W.A. Sirignano, Transient convective burning of interactive fuel droplets in double-layer arrays, Combust. Flame 158 (12) (2011) 2395-2407, http://dx.doi.org/10.1016/j.combustflame.2011.04.011.

[12] G. Castanet, B. Frackowiak, C. Tropea, F. Lemoine, Heat convection within evaporating droplets in strong aerodynamic interactions, Int. J. Heat Mass Transfer 54 (1516) (2011) 3267-3276, http://dx.doi.org/10.1016/j. ijheatmasstransfer.2011.03.060.

[13] W.A. Sirignano, Advances in droplet array combustion theory and modeling, Prog. Energy Combust. Sci. 42 (2014) 54-86, http://dx.doi.org/10.1016/j. pecs.2014.01.002.

[14] L. Perrin, G. Castanet, F. Lemoine, Characterization of the evaporation of interacting droplets using combined optical techniques, Exp. Fluids 56 (2) (2015), http://dx.doi.org/10.1007/s00348-015-1900-3.

[15] P. Lavieille, F. Lemoine, G. Lavergne, M. Lebouché, Evaporating and combusting droplet temperature measurements using two-color laser-induced fluorescence, Exp. Fluids 31 (1) (2001) 45-55, http://dx.doi.org/10.1007/ s003480000257.

[16] F. Lemoine, G. Castanet, Temperature and chemical composition of droplets by optical measurement techniques: a state-of-the-art review, Exp. Fluids 54 (7) (2013), http://dx.doi.org/10.1007/s00348-013-1572-9.

[17] D. Spalding, The combustion of liquid fuels, Symp. (Int.) Combust. 4 (1) (1953) 847-864, http://dx.doi.org/10.1016/S0082-0784(53)80110-4. Fourth Symposium (International) on Combustion.

[18] G. Godsave, Studies of the combustion of drops in a fuel spray the burning of single drops of fuel, Symp. (Int.) Combust. 4 (1) (1953) 818-830, http://dx.doi. org/10.1016/S0082-0784(53)80107-4. Fourth Symposium (International) on Combustion.

[19] G. Castanet, A. Labergue, F. Lemoine, Internal temperature distributions of interacting and vaporizing droplets, Int. J. Therm. Sci. 50 (7) (2011) 1181 1190, http://dx.doi.org/10.1016/j.ijthermalsci.2011.02.001.

[20] S. Sazhin, A. Elwardany, P. Krutitskii, V. Deprédurand, G. Castanet, F. Lemoine, E. Sazhina, M. Heikal, Multi-component droplet heating and evaporation: numerical simulation versus experimental data, Int. J. Therm. Sci. 50 (7) (2011) 1164-1180, http://dx.doi.org/10.1016/j.ijthermalsci.2011.02.020.

[21] M. Labowsky, A formalism for calculating the evaporation rates of rapidly evaporating interacting particles, Combust. Sci. Technol. 18 (3-4) (1978) 145151, http://dx.doi.org/10.1080/00102207808946846.

[22] A. Umemura, Interactive droplet vaporization and combustion: approach from asymptotics, Prog. Energy Combust. Sci. 20 (4) (1994) 325-372, http://dx.doi. org/10.1016/0360-1285(94)90013-2.

[23] R.T. Imaoka W.A Sirignano, Vaporization and combustion in threedimensional droplet arrays, Proc. Combust. Inst. 30 (2) (2005) 1981-1989, http://dx.doi.org/10.1016/j.proci.2004.08.049.

[24] G. Wu, W.A. Sirignano, Transient convective burning of a periodic fuel-droplet array, Proc. Combust. Inst. 33 (2) (2011) 2109-2116, http://dx.doi.org/ 10.1016/j.proci.2010.05.012.

[25] W. Ranz, W. Marshall, Evaporation from drops, Chem. Eng. Prog. 48 (1952) $141-146$.

[26] R. Clift, J.R. Grace, E.M. Weber, Bubbles, Drops, and Particles, Academic Press, New York, London, 1978. 